\title{
INVARIANT HERMITIAN FORMS ON VERTEX ALGEBRAS
}

\author{
VICTOR G. KAC \\ PIERLUIGI MÖSENEDER FRAJRIA \\ PAOLO PAPI
}

\begin{abstract}
We study invariant Hermitian forms on a conformal vertex algebra and on their (twisted) modules. We establish existence of a non-zero invariant Hermitian form on an arbitrary $W$-algebra. We show that for a minimal simple $W$-algebra $W_{k}(\mathfrak{g}, \theta / 2)$ this form can be unitary only when its $\frac{1}{2} \mathbb{Z}$-grading is compatible with parity, unless $W_{k}(\mathfrak{g}, \theta / 2)$ "collapses" to its affine subalgebra.
\end{abstract}

\section{INTRODUCTION}

In the present paper we study invariant Hermitian forms on a conformal vertex algebra $V$ and its (possibly twisted) positive energy modules. By a conformal vertex algebra we mean a vector superspace $V$ over $\mathbb{C}$, endowed with a structure of a vertex algebra (with state-field correspondence $a \mapsto Y(a, z)$ ), and a Virasoro vector $L$ such that the eigenvalues of $L_{0}$ lie in $\frac{1}{2} \mathbb{Z}_{+}$, all eigenspaces are finite-dimensional, and the 0 -th eigenspace consists of multiples of the vacuum vector (cf. Definition 1.1 in Section 2 and [11]).

Let $\phi$ be a conjugate linear involution of $V$. A Hermitian form $(\cdot, \cdot)$ on $V$ is called $\phi$-invariant if, for all $a \in V$, one has

$$
(v, Y(a, z) u)=\left(Y\left(A(z) a, z^{-1}\right) v, u\right), \quad u, v \in V .
$$

Here $A(z): V \rightarrow V((z))$ is defined by

$$
A(z)=e^{z L_{1}} z^{-2 L_{0}} g
$$

where

$$
g(a)=e^{-\pi \sqrt{-1}\left(\frac{1}{2} p(a)+\Delta_{a}\right)} \phi(a), \quad a \in V,
$$

and $p(a)=0$ or 1 stands for the parity of $a$ and $\Delta_{a}$ for its $L_{0}$-eigenvalue. The definition of a $\phi$-invariant Hermitian form on a $V$-module $M$ is similar (cf. Definition 6.6).

The operator $A(z)$ with $g=(-1)^{L_{0}}$ appeared first in [4] in the construction of the coadjoint module in the case when $V$ is purely even and the eigenvalues of $L_{0}$ are integers. Under the same assumptions on $V$ this operator was used in [9] for the construction of the dual to the $V$-modules.

Formula (1.2) with $g=(-1)^{L_{0}} \phi$ was used in [7] to define unitary structures on vertex operator algebras and this notion was generalized in [3] to vertex algebras with $\frac{1}{2} \mathbb{Z}_{+}$-grading compatible with parity, in which case formula (1.3) simplifies to (see (4.2))

$$
g=(-1)^{L_{0}+2 L_{0}^{2}} \phi
$$

As one can infer from the above remarks, the motivation for this definition stems from the observation that, given a $V$-module $M$, one has (as in the Lie algebra case), a bijective correspondence between $\phi$-invariant Hermitian forms $(\cdot, \cdot)$ on $V$ and $V$-module conjugate 
linear homomorphisms $\Theta: M \rightarrow M^{\dagger}$, where $M^{\dagger}$ is the conjugate linear dual to $M$, with $V$-module structure defined by

$$
\left\langle Y_{M^{\dagger}}(a . z) m^{\prime}, m\right\rangle=\left\langle m^{\prime}, Y_{M}\left(A(z) a, z^{-1}\right) m\right\rangle, \quad m \in M, m^{\prime} \in M^{\dagger} .
$$

Our first result, which generalizes [9, Theorem 5.2.1, Proposition 5.3.1] (with a similar proof), is Proposition 3.6. formula (1.4) indeed defines a structure of a $V$-module on the restricted dual superspace $M^{\dagger}$ of $M$. Our second result, which generalizes, with the same proof, that of [15] in the symmetric case, is Proposition 4.3, which describes $\phi$-invariant Hermitian forms on $V$. Its Corollary 4.7 claims that a conformal vertex algebra $V$ with a conjugate linear involution $\phi$ admits a (unique, up to a constant factor) $\phi$-invariant Hermitian form if and only if any eigenvector of $L_{0}$ with eigenvalue 1 is annihilated by $L_{1}$ (see also Remark 4.4). As usual, such a Hermitian form can be expressed in terms of the expectation value on the vacuum (see formula (4.9)).

In Section 5 we construct invariant Hermitian forms of fermionic, bosonic, affine and lattice vertex algebras. In Section [6 we extend the results on invariant Hermitian forms on $V$ to arbitrary positive-energy (twisted) modules $M$. Proposition 5.3 claims that the space of $\phi$-invariant Hermitian forms on $M$ is isomorphic to the set of $\omega$-invariant Hermitian forms on the module $M_{0}$ over the Zhu algebra. Here $M_{0}$ is the lowest energy subspace of $M$ and $\omega$ is the conjugate linear anti-involution of the Zhu algebra, induced by the endomorphism of the superspace $V$ defined by

$$
\omega(v)=A(1) v, \quad v \in V .
$$

In Remark 6.8 we note that actually Proposition 4.3 is a special case of Proposition 6.7.

In Section 7 we construct an invariant Hermitian form on the $W$-algebras $W^{k}(\mathfrak{g}, x, f)$ [12, [13]. This construction is based on Proposition 7.4 (b), which says that the condition

of Corollary 4.7, that all eigenvectors of $L_{0}$ with eigenvalue 1 of the vertex algebra are annihilated by $L_{1}$, holds, provided that the elements $h:=2 x$ and $f$ can be included in a sl(2)-triple $\{e, f, h\}$.

In conclusion of this section we briefly discuss unitarity (i.e., positive semi-definiteness) of this Hermitian form for minimal $W$-algebras $W^{k}(\mathfrak{g}, \theta / 2)$. We show that the only interesting cases might occur when the $\frac{1}{2} \mathbb{Z}$-grading on the $W$-algebra is compatible with parity. In all the other cases we show that the $W$-algebra can be unitary only at collapsing levels [1], i.e. when the simple $W$-algebra $W_{k}(\mathfrak{g}, \theta / 2)$ "collapses" to its affine subalgebra: see Propositions 7.9, 7.11. These are just the first steps towards classification of unitary minimal $W$-algebras.

Throughout the paper the base field is $\mathbb{C}$. We also denote by $\mathbb{Z}_{+}$the set of nonnegative integers and by $\mathbb{N}$ the set of positive integers.

\section{Setup}

2.1. Basic definitions. Recall that a vector superspace is a $\mathbb{Z} / 2 \mathbb{Z}$-graded vector space $V=$ $V_{\overline{0}} \oplus V_{\overline{1}}$. The elements in $V_{\overline{0}}\left(\right.$ resp. $\left.V_{\overline{1}}\right)$ are called even (resp. odd). Set

$$
p(v)= \begin{cases}0 \in \mathbb{Z} & \text { if } v \in V_{\overline{0}}, \\ 1 \in \mathbb{Z} & \text { if } v \in V_{\overline{1}},\end{cases}
$$

i.e. we will regard $p(v)$ as an integer, not as a residue class. We will often use the notation

$$
\sigma(u)=(-1)^{p(u)} u, \quad p(u, v)=(-1)^{p(u) p(v)} .
$$


Let $V$ be a vertex algebra. We let

$$
\begin{aligned}
& Y: V \rightarrow(\text { End } V)\left[\left[z, z^{-1}\right]\right], \\
& v \mapsto Y(v, z)=\sum_{n \in \mathbb{Z}} v_{(n)} z^{-n-1} \quad\left(v_{(n)} \in \text { End } V\right),
\end{aligned}
$$

be the state-field correspondence. We denote by 1 the vacuum vector in $V$ and by $T$ the translation operator (see e.g. [11] for details).

Definition 2.1. In the present paper we will call a vertex algebra $V$ conformal if there exists a distinguished vector $L \in V_{2}$, called a Virasoro vector, satisfying the following conditions:

$$
\begin{aligned}
& Y(L, z)=\sum_{n \in \mathbb{Z}} L_{n} z^{-n-2},\left[L_{m}, L_{n}\right]=(m-n) L_{m+n}+\frac{1}{12}\left(m^{3}-m\right) \delta_{m+n, 0} c I, \\
& L_{-1}=T, \\
& L_{0} \text { is diagonalizable and its eigenspace decomposition has the form }
\end{aligned}
$$

$$
V=\bigoplus_{n \in \frac{1}{2} \mathbb{Z}_{+}} V_{n}
$$

where

$$
\operatorname{dim} V_{n}<\infty \text { for all } n \text { and } V_{0}=\mathbb{C} \mathbf{1} .
$$

The number $c$ is called the central charge.

Remark 2.2. Important examples of conformal vertex algebras are vertex operator superalgebras, namely the conformal vertex algebras for which decomposition (2.6) is compatible with parity, i.e. $\sigma(u)=(-1)^{2 L_{0}} u$.

In the definition of [11] of conformal vertex algebras properties (2.6) and (2.7) are not required.

By an automorphism of a conformal vertex algebra $V$ we mean a vertex algebra automorphism $\phi$ of $V$ (i. e. $\phi\left(u_{(n)} v\right)=\phi(u)_{(n)} \phi(v)$ for all $\left.n \in \mathbb{Z}\right)$ with the property that $\phi(L)=L$. Consequently, $\phi\left(V_{n}\right)=V_{n}$.

The eigenvalues of $L_{0}$ on $V$ are called conformal weights; the conformal weight of $v \in V$ is denoted by $\Delta_{v}$, so that $v \in V_{\Delta_{v}}$. The eigenvector $v$ of $L_{0}$ is called quasiprimary if $L_{1} v=0$ and primary if $L_{n} v=0$ for $n \geq 1$. One has for $v$ of conformal weight $\Delta_{v}$ :

$$
\left[L_{\lambda} v\right]=\left(L_{-1}+\Delta_{v} \lambda\right) v+\sum_{n \geq 2} \frac{\lambda^{n}}{n !} L_{n-1} v .
$$

Here and throughout the paper we use the formalism of $\lambda$-brackets, which are defined by

$$
\left[u_{\lambda} v\right]=\operatorname{Res}_{z} e^{z \lambda} Y(u, z) v, \quad u, v \in V .
$$

Let $\Gamma$ be an additive subgroup of $\mathbb{R}$ containing $\mathbb{Z}$. If $\gamma \in \mathbb{R}$, denote by $[\gamma]$ its coset $\gamma+\mathbb{Z}$.

Definition 2.3. Let $V$ be a conformal vertex algebra. A $\Gamma / \mathbb{Z}$-grading on $V$ is a map $\Upsilon:[\gamma] \mapsto V^{[\gamma]} \subseteq V$ such that $V$ decomposes as

$$
V=\bigoplus_{[\gamma] \in \Gamma / \mathbb{Z}} V^{[\gamma]}
$$

and (2.9) is a vertex algebra grading, compatible with $L_{0}$, i.e.

$$
V_{(n)}^{[\alpha]} V^{[\beta]} \subseteq V^{[\alpha+\beta]}, \quad L_{0}\left(V^{[\gamma]}\right) \subseteq V^{[\gamma]} .
$$


If $a \in V^{[\gamma]}$ then $[\gamma]$ is called the degree of $a$. Given a vector $a \in V$ of conformal weight $\Delta_{a}$ and degree $[\gamma]$, denote by $\epsilon_{a}$ the maximal non-positive real number in the coset $\left[\gamma-\Delta_{a}\right]$. This number has the following properties [5]:

$$
\epsilon_{\mathbf{1}}=0, \quad \epsilon_{T a}=\epsilon_{a}, \quad \epsilon_{a_{(n)} b}=\epsilon_{a}+\epsilon_{b}+\chi(a, b),
$$

where $\chi(a, b)=1$ or 0 , depending on whether $\epsilon_{a}+\epsilon_{b} \leq-1$ or not.

Let $\gamma_{a}=\Delta_{a}+\epsilon_{a}$. Then

$$
\gamma_{\mathbf{1}}=0, \quad \gamma_{T a}=\gamma_{a}+1, \quad \gamma_{a_{(n)} b}=\gamma_{a}+\gamma_{b}+\chi(a, b)-n-1 .
$$

\subsection{Twisted modules.}

Definition 2.4. Let $\Gamma$ be an additive subgroup of $\mathbb{R}$ containing $\mathbb{Z}$, and let $\Upsilon$ be a $\Gamma / \mathbb{Z}$-grading on a conformal vertex algebra $V$. A $\Upsilon$-twisted module for $V$ is a vector superspace $M$ and a parity preserving linear map from $V$ to the space of End $M$-valued $\Upsilon$-twisted quantum fields $a \mapsto Y^{M}(a, z)=\sum_{m \in\left[\gamma_{a}\right]} a_{(m)}^{M} z^{-m-1}$ (i.e. $a_{(m)}^{M} \in \operatorname{End} M$ and $a_{(m)}^{M} v=0$ for each $v \in M$ and $m \gg 0)$, such that the following properties hold:

$$
\begin{gathered}
\mathbf{1}_{(n)}^{M}=\delta_{n,-1} I_{M}, \\
\sum_{j \in \mathbb{Z}_{+}}\left(\begin{array}{c}
m \\
j
\end{array}\right)\left(a_{(n+j)} b\right)_{(m+k-j)}^{M} v \\
=\sum_{j \in \mathbb{Z}_{+}}(-1)^{j}\left(\begin{array}{c}
n \\
j
\end{array}\right)\left(a_{(m+n-j)}^{M} b_{(k+j)}^{M}-p(a, b)(-1)^{n} b_{(k+n-j)}^{M} a_{(m+j)}^{M}\right) v,
\end{gathered}
$$

where $a \in V^{\left[\gamma_{a}\right]}\left(\gamma_{a} \in \Gamma\right), m \in\left[\gamma_{a}\right], n \in \mathbb{Z}, k \in\left[\gamma_{b}\right]$.

The following Lemma is known; we prove it for completeness.

Lemma 2.5. The Borcherds identity (2.13) is equivalent to

$$
\begin{aligned}
& \operatorname{Res}_{u}\left(i_{w, u} Y_{M}(Y(a, u) b, w)(w+u)^{m} u^{n} w^{l}\right)= \\
& \operatorname{Res}_{z}\left(i_{z, w} Y_{M}(a, z) Y_{M}(b, w) z^{m}(z-w)^{n} w^{l}-p(a, b) i_{w, z} Y_{M}(b, w) Y_{M}(a, z) z^{m}(z-w)^{n} w^{l}\right)
\end{aligned}
$$

for all $n \in \mathbb{Z}, m \in\left[\gamma_{a}\right], l \in\left[\gamma_{b}\right]$. As usual, $i_{x, y}$ means expanding in the domain $|x|>|y|$.

Proof. Computing the residues we find that (2.14) is equivalent to

$$
\begin{aligned}
& \sum_{t \in \mathbb{Z}, j \in \mathbb{Z}_{+}}\left(\begin{array}{c}
m \\
j
\end{array}\right)\left(a_{(j+n)} b\right)_{(t-j+m+l)}^{M} w^{-t-1} \\
= & \left.\sum_{t \in \mathbb{Z}, j \in \mathbb{Z}_{+}}(-1)^{j}\left(\begin{array}{c}
n \\
j
\end{array}\right)\left(a_{(m+n-j)}^{M} b_{(t+j+l)}^{M}-p(a, b)(-1)^{n} b_{(t+n-j+l)}^{M} a_{(m+j)}^{M}\right)\right) w^{-t-1} .
\end{aligned}
$$

Since $V^{[\gamma]}$ is $L_{0}$-invariant, we have its eigenspace decomposition $V^{[\gamma]}=\oplus_{\Delta} V_{\Delta}^{[\gamma]}$, and we will write for $v \in V_{\Delta_{v}}^{[\gamma]}$,

$$
Y_{M}(v, z)=\sum_{n \in\left[\gamma-\Delta_{v}\right]} v_{n}^{M} z^{-n-\Delta_{v}}
$$


Definition 2.6. A $\Upsilon$-twisted $V$-module $M$ is called a positive energy $V$-module if $M$ has an $\mathbb{R}$-grading $M=\oplus_{j \geq 0} M_{j}$ such that

$$
a_{n}^{M} M_{j} \subseteq M_{j-n}, a \in V_{\Delta_{a}} .
$$

The subspace $M_{0}$ is called the minimal energy subspace. Then,

$$
a_{n}^{M} M_{0}=0 \text { for } n>0 \text { and } a_{0}^{M} M_{0} \subseteq M_{0} .
$$

2.3. Zhu algebras. Set

$$
V_{\Upsilon}=\operatorname{span}\left(a \in V \mid \epsilon_{a}=0\right) .
$$

Define a subspace $J_{\Upsilon}$ of $V$ as the span of elements

$$
\sum_{j \in \mathbb{Z}_{+}}\left(\begin{array}{c}
\gamma_{a} \\
j
\end{array}\right) a_{(-2+\chi(a, b)+j)} b=\operatorname{Res}_{z} z^{-2+\chi(a, b)} Y\left((1+z)^{\gamma_{a}} a, z\right) b,
$$

with $\epsilon_{a}+\epsilon_{b} \in \mathbb{Z}$.

Let

$$
a * b=\sum_{j \in \mathbb{Z}_{+}}\left(\begin{array}{c}
\gamma_{a} \\
j
\end{array}\right) a_{(-1+j)} b,
$$

Then $J_{\Upsilon}$ is a two sided ideal in $V_{\Upsilon}$ with respect to the product $*$. The quotient $Z h u \Upsilon(V)=$ $V_{\Upsilon} / J_{\Upsilon}$ is an associative superalgebra with respect to the product $*$ (see [5] for a proof), which is called the Zhu algebra associated to the grading (2.9).

Example 2.7. If $\Gamma$ is the subgroup of $\mathbb{R}$ spanned by the conformal weights $\Delta_{a}$ then one has a $\Gamma / \mathbb{Z}$-grading (2.9), for which

$$
V^{[\gamma]}=\oplus_{\Delta \in[\gamma]} V_{\Delta} .
$$

The corresponding Zhu algebra is called the $L_{0}$-twisted (or Ramond twisted) Zhu algebra and denoted by $Z h u_{L_{0}} V$. If $\Gamma=\mathbb{Z}$ then one has the trivial grading (2.9) by setting $V^{\mathbb{Z}}=V$. The corresponding Zhu algebra is denoted by $Z h u_{\mathbb{Z}} V$ and is called the non-twisted Zhu algebra ([5], Examples 2.14 and 2.15).

\section{The Conjugate Contragredient module}

In this section we adapt to our setting the proofs of Section 5 of [9], where the action of a vertex operator algebra on the linear dual of a module is defined. If $a \in V_{\Delta_{a}}$, set

$$
(-1)^{L_{0}} a=e^{\pi \sqrt{-1} \Delta_{a}} a, \quad \sigma^{1 / 2}(a)=e^{\frac{\pi}{2} \sqrt{-1} p(a)} a .
$$

Lemma 3.1. Let $g$ be a diagonalizable parity preserving conjugate linear operator on $V$ with modulus 1 eigenvalues, such that $g(L)=L$. Then one has the relation

$$
g Y(a, z) g^{-1} b=p(a, b) Y(g(a),-z) b
$$

if and only if the operator

$$
\phi=(-1)^{L_{0}} \sigma^{1 / 2} g
$$

is a conjugate linear automorphism of $V$. Moreover

$$
g^{2}=I \Longleftrightarrow \phi^{2}=I \text {. }
$$


Proof. Assume that $g$ satisfies (3.2). Then

$$
\begin{aligned}
\phi(a)_{(n)} \phi(b) & =\left((-1)^{L_{0}} \sigma^{1 / 2} g\right)(a)_{(n)}\left((-1)^{L_{0}} \sigma^{1 / 2} g\right)(b) \\
& =e^{\pi \sqrt{-1}\left(\Delta_{a}+\Delta_{b}\right)} e^{\pi / 2 \sqrt{-1}(p(a)+p(b))} g(a)_{(n)} g(b) .
\end{aligned}
$$

By (3.2), $g\left(a_{(n)} b\right)=(-1)^{n+1} p(a, b) g(a)_{(n)} g(b)$. Substituting in (3.5), and noting that $p(a)+$ $p(b)+2 p(a) p(b)=p\left(a_{(n)} b\right) \bmod 4 \mathbb{Z}$, we obtain

$$
\begin{aligned}
\phi(a)_{(n)} \phi(b) & =e^{\pi \sqrt{-1}\left(\Delta_{a}+\Delta_{b}\right)} e^{\pi / 2 \sqrt{-} 1(p(a)+p(b))}(-1)^{n+1} p(a, b) g\left(a_{(n)} b\right) \\
& =e^{\pi \sqrt{-1} \Delta_{a_{(n)}} b} e^{\pi / 2 \sqrt{-1} 1(p(a)+p(b)+2 p(a) p(b))} g\left(a_{(n)} b\right) \\
& =e^{\pi \sqrt{-1} \Delta_{a_{(n)}} b} e^{\pi / 2 \sqrt{-} 1 p\left(a_{(n)} b\right)} g\left(a_{(n)} b\right)=\phi\left(a_{(n)} b\right) .
\end{aligned}
$$

Reversing the argument we obtain the converse statement.

To prove (3.4) remark that $g(L)=L$, hence $L_{0} g(a)=g(L)_{0} g(a)=g\left(L_{0} a\right)$, so, since $\Delta_{a} \in \mathbb{R}, \Delta_{g(a)}=\Delta_{a}$. Moreover $g$ is parity preserving and conjugate linear, hence

$$
\begin{aligned}
\phi^{2}(a) & =(-1)^{L_{0}} \sigma^{1 / 2} g(-1)^{L_{0}} \sigma^{1 / 2} g(a)=e^{\pi \sqrt{-1}\left(\Delta_{a}+\frac{1}{2} p(a)\right)} g e^{\pi \sqrt{-1}\left(\Delta_{a}+\frac{1}{2} p(a)\right)} g(a) \\
& =e^{\pi \sqrt{-1}\left(\Delta_{a}+\frac{1}{2} p(a)\right)} e^{-\pi \sqrt{-1}\left(\Delta_{a}+\frac{1}{2} p(a)\right)} g^{2}(a)=g^{2}(a) .
\end{aligned}
$$

Definition 3.2. Let $g$ be a diagonalizable parity preserving conjugate linear operator on $V$, satisfying (3.2) and such that $g^{2}=I$. Define $A(z): V \rightarrow V((z))$ by

$$
A(z) v=e^{z L_{1}} z^{-2 L_{0}} g v, \quad v \in V \text {. }
$$

Lemma 3.3. We have

$$
p(a, b) A(w) Y(a, z) A(w)^{-1} b=i_{w, z} Y\left(A(z+w) a, \frac{-z}{(z+w) w}\right) b
$$

and

$$
A\left(z^{-1}\right)=A(z)^{-1} \text {. }
$$

Proof. It is clear that

$$
w^{-2 L_{0}} Y(a, z) w^{2 L_{0}} b=Y\left(w^{-2 L_{0}} a, z / w^{2}\right) b
$$

By (3.2)

$$
p(a, b) g w^{-2 L_{0}} Y(a, z) w^{2 L_{0}} g^{-1} b=Y\left(g w^{-2 L_{0}} a,-z / w^{2}\right) b .
$$

Finally we use that, if $|w z|<1$, then

$$
e^{w L_{1}} Y(a, z) e^{-w L_{1}}=Y\left(e^{w(1-w z) L_{1}}(1-w z)^{-2 L_{0}} a, \frac{z}{1-w z}\right)
$$

(see (5.2.38) of [9] and (4.9.17) of [11]) to get, for $|z|<|w|$,

$$
p(a, b) e^{w L_{1}} Y\left(g w^{-2 L_{0}} a,-z / w^{2}\right) e^{-w L_{1}} b=Y\left(e^{(w+z) L_{1}} g(w+z)^{-2 L_{0}} a, \frac{-z}{w(w+z)}\right) b,
$$

which is 3.77).

Since $g^{2}=I$, (3.8) is equivalent to

$$
A(z) a=g^{-1} z^{-2 L_{0}} e^{-z^{-1} L_{1}} a=g z^{-2 L_{0}} e^{-z^{-1} L_{1}} a .
$$


Next observe that

$$
\begin{aligned}
g z^{-2 L_{0}} e^{-z^{-1} L_{1}} a & =\sum_{r} z^{-2 L_{0}}(-1)^{r} \frac{1}{r !} g\left(L_{1}^{r} a\right) z^{-r} \\
& =\sum_{r} z^{-2 \Delta_{a}} z^{2 r}(-1)^{r} \frac{1}{r !} g\left(L_{1}^{r} a\right) z^{-r} \\
& =\sum_{r}(-1)^{r} \frac{1}{r !} g\left(L_{1}^{r} a\right) z^{r-2 \Delta_{a}} .
\end{aligned}
$$

Since $g\left(L_{1} v\right)=-g(L)_{1} g(v)=-L_{1} g(v)$ we obtain

$$
g z^{-2 L_{0}} e^{-z^{-1} L_{1}} a=\sum_{r} \frac{1}{r !} L_{1}^{r} g(a) z^{r-2 \Delta_{a}}=e^{z L_{1}} z^{-2 L_{0}} g(a)=A(z) a .
$$

Remark 3.4. Note that, by (3.13), if $v$ is quasiprimary, we have

$$
A(z) v=z^{-2 \Delta_{v}} g(v) .
$$

If $\Upsilon$ is a $\Gamma / \mathbb{Z}$-grading on $V$, we let the opposite grading $-\Upsilon$ be the grading defined by setting

$$
-\Upsilon([\gamma])=\Upsilon(-[\gamma])
$$

We say that a $\Gamma / \mathbb{Z}$-grading is compatible with a map $\phi$ if $\phi\left(V^{[\gamma]}\right) \subseteq V^{[\gamma]}$.

Let $M$ be a positive energy $\Upsilon$-twisted module and let $M^{\dagger}$ denote the restricted conjugate dual of $M$, that is

$$
M^{\dagger}=\bigoplus_{n \geq 0} M_{n}^{\dagger}
$$

where $M_{n}^{\dagger}$ is the space of conjugate linear maps from $M_{n}$ to $\mathbb{C}$.

Lemma 3.5. If $M+K \in \mathbb{Z}$, then

$$
\operatorname{Res}_{z} z^{M} w^{N} i_{z, w}(z+w)^{K}=(-1)^{K+M-1} \operatorname{Res}_{z} z^{-2-K-M} w^{2+2 K+M+N} i_{w, z}(z+w)^{M} .
$$

Proof. If $M+K<-1$, both sides of (3.16) are zero. If $M+K \geq-1$, then

$$
\begin{aligned}
\operatorname{Res}_{z} z^{M} w^{N} i_{z, w}(z+w)^{K} & =\operatorname{Res}_{z} \sum_{j \in \mathbb{Z}_{+}}\left(\begin{array}{c}
K \\
j
\end{array}\right) z^{M+K-j} w^{N+j} \\
& =\left(\begin{array}{c}
K \\
M+K+1
\end{array}\right) w^{N+M+K+1} .
\end{aligned}
$$

On the other hand

$$
\begin{aligned}
\operatorname{Res}_{z} z^{m} w^{n} i_{w, z}(z+w)^{k} & =\operatorname{Res}_{z} \sum_{j \in \mathbb{Z}_{+}}\left(\begin{array}{l}
k \\
j
\end{array}\right) z^{m+j} w^{n+k-j} \\
& =\left(\begin{array}{c}
k \\
-m-1
\end{array}\right) w^{n+m+k+1} \\
& =(-1)^{-m-1}\left(\begin{array}{c}
-m-1-k-1 \\
-m-1
\end{array}\right) w^{n+m+k+1} .
\end{aligned}
$$

Equality holds for $m=-2-K-M, n=2+2 K+M+N, k=M$. 
Theorem 3.6. Let $\phi$ be a conjugate linear involution of a conformal vertex algebra $V$. Choose $g$ as in Definition 3.2 and define $A(z)$ by (3.6). Let $\Upsilon$ be a $\Gamma / \mathbb{Z}$-grading on $V$ compatible with $\phi$. Let $M$ be a $\Upsilon$-twisted positive energy module. Then

(a) The map $Y_{M^{\dagger}}$ given by

$$
\left\langle Y_{M^{\dagger}}(v, z) m^{\prime}, m\right\rangle=\left\langle m^{\prime}, Y_{M}\left(A(z) v, z^{-1}\right) m\right\rangle, m \in M, m^{\prime} \in M^{\dagger},
$$

defines on $M^{\dagger}$ the structure of a $(-\Upsilon)$-twisted $V$-module.

(b) If $\operatorname{dim} M_{n}<\infty$ for all $n$ then $\left(M^{\dagger}\right)^{\dagger}$ is naturally isomorphic to $M$.

Proof. Let $V=\oplus_{\gamma \in \Gamma / \mathbb{Z}} V^{\gamma}$ be the grading $\Upsilon$. Write explicitly for $v \in V_{\Delta_{v}}^{\gamma}$,

$$
Y_{M^{\dagger}}(v, z)=\sum_{n \in-\gamma-\Delta_{v}} v_{n}^{M^{\dagger}} z^{-n-\Delta_{v}} .
$$

Then we have

$$
\sum_{n}\left\langle v_{n}^{M^{\dagger}} m^{\prime}, m\right\rangle z^{-n-\Delta_{v}}=\sum_{n}\left\langle m^{\prime}, \sum_{t} \frac{1}{t !}\left(L_{1}^{t} g(v)\right)_{n}^{M} m\right\rangle z^{n-\Delta_{v}} .
$$

In other words, if $n \in-\gamma-\Delta_{v}$, then

$$
\left\langle v_{n}^{M^{\dagger}} m^{\prime}, m\right\rangle=\left\langle m^{\prime}, \sum_{t} \frac{1}{t !}\left(L_{1}^{t} g(v)\right)_{-n}^{M} m\right\rangle .
$$

In particular, $v_{n}^{M^{\dagger}} M_{j}^{\dagger} \subseteq M_{j-n}^{\dagger}$. This proves that, by (3.15), $Y^{M^{\dagger}}$ is indeed a $(-\Upsilon)$-twisted quantum field.

Next observe that

$$
\left\langle\mathbf{1}_{(n)}^{M^{\dagger}} m^{\prime}, m\right\rangle=\left\langle\mathbf{1}_{n+1}^{M^{\dagger}} m^{\prime}, m\right\rangle=\left\langle m^{\prime}, \mathbf{1}_{-n-1}^{M} m\right\rangle=\delta_{-n-1,0}\left\langle m^{\prime}, m\right\rangle,
$$

hence (2.12) for $M^{\dagger}$ follows.

We now prove the Borcherds identity (2.14) for $M^{\dagger}$, that is

$$
\begin{aligned}
& \operatorname{Res}_{u}\left\langle Y_{M^{\dagger}}(Y(a, u) b, w) i_{w, u}(w+u)^{k} u^{n} w^{l} m^{\prime}, m\right\rangle \\
& =\operatorname{Res}_{z}\left(\left\langle Y_{M^{\dagger}}(a, z) Y_{M^{\dagger}}(b, w) i_{z, w} z^{k}(z-w)^{n} w^{l} m^{\prime}, m\right\rangle\right) \\
& -p(a, b) \operatorname{Res}_{z}\left(\left\langle Y_{M^{\dagger}}(b, w) Y_{M^{\dagger}}(a, z) i_{w, z} z^{k}(z-w)^{n} w^{l} m^{\prime}, m\right\rangle\right)
\end{aligned}
$$

for all $n \in \mathbb{Z}, k \in\left[-\gamma_{a}\right], l \in\left[-\gamma_{b}\right]$. Since

$$
\begin{aligned}
\left\langle Y_{M^{\dagger}}(a, z) Y_{M^{\dagger}}(b, w) m^{\prime}, m\right\rangle & =\left\langle m^{\prime}, Y_{M}\left(A(w) b, w^{-1}\right) Y_{M}\left(A(z) a, z^{-1}\right) m\right\rangle, \\
\left\langle Y_{M^{\dagger}}(b, w) Y_{M^{\dagger}}(a, z) m^{\prime}, m\right\rangle & =\left\langle m^{\prime}, Y_{M}\left(A(z) a, z^{-1}\right) Y_{M}\left(A(w) b, w^{-1}\right) m\right\rangle, \\
\left\langle Y_{M^{\dagger}}(Y(a, u) b, w) m^{\prime}, m\right\rangle & =\left\langle m^{\prime}, Y_{M}\left(A(w) Y(a, u) b, w^{-1}\right) m\right\rangle,
\end{aligned}
$$

we have to prove that

$$
\begin{aligned}
& \operatorname{Res}_{u}\left(\left\langle m^{\prime}, Y_{M}\left(A(w) Y(a, u) b, w^{-1}\right) m\right\rangle i_{w, u}(w+u)^{k} u^{n} w^{l}\right) \\
& =\operatorname{Res}_{z}\left(\left\langle m^{\prime}, Y_{M}\left(A(w) b, w^{-1}\right) Y_{M}\left(A(z) a, z^{-1}\right) m\right\rangle i_{z, w} z^{k}(z-w)^{n} w^{l}\right) \\
& -p(a, b) \operatorname{Res}_{z}\left(\left\langle m^{\prime}, Y_{M}\left(A(z) a, z^{-1}\right) Y_{M}\left(A(w) b, w^{-1}\right) m\right\rangle i_{w, z} z^{k}(z-w)^{n} w^{l}\right) .
\end{aligned}
$$

Hence we need to check that

$$
\begin{aligned}
& \operatorname{Res}_{u}\left(Y_{M}\left(A(w) Y(a, u) b, w^{-1}\right) i_{w, u}(w+u)^{k} u^{n} w^{l}\right) \\
& =\operatorname{Res}_{z}\left(Y_{M}\left(A(w) b, w^{-1}\right) Y_{M}\left(A(z) a, z^{-1}\right) i_{z, w} z^{k}(z-w)^{n} w^{l}\right) \\
& -p(a, b) \operatorname{Res}_{z}\left(Y_{M}\left(A(z) a, z^{-1}\right) Y_{M}\left(A(w) b, w^{-1}\right) i_{w, z} z^{k}(z-w)^{n} w^{l}\right) .
\end{aligned}
$$


Changing variables in the Borcherds identity (2.14) for $Y_{M}$ we obtain, for all $n \in \mathbb{Z}, m \in\left[\gamma_{a}\right]$, $l \in\left[\gamma_{b}\right]$,

$$
\begin{aligned}
& \operatorname{Res}_{t} Y_{M}\left(Y\left(a, t^{-1}\right) b, w^{-1}\right) i_{w^{-1}, t^{-1}}\left(w^{-1}+t^{-1}\right)^{m} t^{-n-2} w^{-l} \\
& =\operatorname{Res}_{t}\left(Y_{M}\left(a, t^{-1}\right) Y_{M}\left(b, w^{-1}\right) i_{t^{-1}, w^{-1}} t^{-m-2}\left(t^{-1}-w^{-1}\right)^{n} w^{-l}\right) \\
& -p(a, b) \operatorname{Res}_{t}\left(Y_{M}\left(b, w^{-1}\right) Y_{M}\left(a, t^{-1}\right) i_{w^{-1}, t^{-1}} t^{-m-2}\left(t^{-1}-w^{-1}\right)^{n} w^{-l}\right),
\end{aligned}
$$

which is equivalent to

$$
\begin{aligned}
& \operatorname{Res}_{t}\left(Y_{M}\left(Y\left(a, t^{-1}\right) b, w^{-1}\right) i_{t, w}(w+t)^{m} t^{-n-2-m} w^{-l-m}\right. \\
& =\operatorname{Res}_{t}\left(Y_{M}\left(a, t^{-1}\right) Y_{M}\left(b, w^{-1}\right) i_{w, t} t^{-m-n-2}(w-t)^{n} w^{-l-n}\right) \\
& -p(a, b) \operatorname{Res}_{t}\left(Y_{M}\left(b, w^{-1}\right) Y_{M}\left(a, t^{-1}\right) i_{t, w} t^{-m-2-n}(w-t)^{n} w^{-l-n}\right) .
\end{aligned}
$$

Write explicitly $A(w) a=\sum_{r \in \mathbb{Z}_{+}} C_{r}(a) w^{r-2 \Delta_{a}}$, where $C_{r}(a) \in V$. Then

$$
Y_{M}\left(A(t) a, t^{-1}\right)=\sum_{r \in \mathbb{Z}_{+}, h \in\left[\gamma_{a}\right]} C_{r}(a)_{(h)} t^{h+1} t^{r-2 \Delta_{a}}=\sum_{r \in \mathbb{Z}_{+}} Y_{M}\left(C_{r}(a), t^{-1}\right) t^{r-2 \Delta_{a}},
$$

so, by (3.22),

$$
\begin{aligned}
& \operatorname{Res}_{t}\left(Y_{M}\left(A(t) a, t^{-1}\right) Y_{M}\left(A(w) b, w^{-1}\right) i_{w, t} t^{-m-n-2}(w-t)^{n} w^{-l-n}\right) \\
& -p(a, b) \operatorname{Res}_{t}\left(Y_{M}\left(A(w) b, w^{-1}\right) Y_{M}\left(A(t) a, t^{-1}\right) i_{t, w} t^{-m-2-n}(w-t)^{n} w^{-l-n}\right) \\
& =\sum_{r} \operatorname{Res}_{t}\left(Y_{M}\left(C_{r}(a), t^{-1}\right) Y_{M}\left(A(w) b, w^{-1}\right) i_{w, t} t^{-m-n-2+r-2 \Delta_{a}}(w-t)^{n} w^{-l-n}\right) \\
& -p(a, b) \sum_{r} \operatorname{Res}_{t}\left(Y_{M}\left(A(w) b, w^{-1}\right) Y_{M}\left(C_{r}(a), t^{-1}\right) i_{t, w} t^{-m-2-n+r-2 \Delta_{a}}(w-t)^{n} w^{-l-n}\right) \\
& =\sum_{r} \operatorname{Res}_{t}\left(Y_{M}\left(Y\left(C_{r}(a), t^{-1}\right) A(w) b, w^{-1}\right) i_{t, w}(w+t)^{m-r+2 \Delta_{a}} t^{-n-2-m+r-2 \Delta_{a}} w^{-l-m+r-2 \Delta_{a}}\right. \\
& =\operatorname{Res}_{t}\left(Y_{M}\left(i_{t, w} Y\left(A\left(\frac{w t}{w+t}\right) a, t^{-1}\right) A(w) b, w^{-1}\right)(w+t)^{m} t^{-n-2-m} w^{-l-m} .\right.
\end{aligned}
$$

Therefore we have

$$
\begin{aligned}
& \operatorname{Res}_{z}\left(Y_{M}\left(A(z) a, z^{-1}\right) Y_{M}\left(A(w) b, w^{-1}\right) i_{w, z} z^{k}(w-z)^{n} w^{l}\right) \\
& -p(a, b) \operatorname{Res}_{z}\left(Y_{M}\left(A(w) b, w^{-1}\right) Y_{M}\left(A(z) a, z^{-1}\right) i_{z, w} z^{k}(w-z)^{n} w^{l}\right) \\
& =\operatorname{Res}_{t}\left(Y_{M}\left(i_{t, w} Y\left(A\left(\frac{w t}{w+t}\right) a, t^{-1}\right) A(w) b, w^{-1}\right)(w+t)^{-k-n-2} t^{k} w^{l+k+2 n+2} .\right.
\end{aligned}
$$

Hence (3.21) turns into

$$
\begin{aligned}
& \operatorname{Res}_{t}\left(i_{t, w} Y_{M}\left(Y\left(A\left(\frac{w t}{w+t}\right) a, t^{-1}\right) A(w) b, w^{-1}\right)(w+t)^{-k-n-2} t^{k} w^{l+k+2 n+2}\right. \\
& =-p(a, b)(-1)^{n} \operatorname{Res}_{t}\left(Y_{M}\left(A(w) Y(a, t) b, w^{-1}\right) i_{w, t}(w+t)^{k} t^{n} w^{l}\right) .
\end{aligned}
$$

Expand the L.H.S. above as

$$
\begin{aligned}
& \operatorname{Res}_{t}\left(Y_{M}\left(i_{t, w} Y\left(A\left(\frac{w t}{w+t}\right) a, t^{-1}\right) A(w) b, w^{-1}\right)(w+t)^{-k-n-2} t^{k} w^{l+k+2 n+2}\right)= \\
& \sum_{p, q, r, s} \operatorname{Res}_{t}\left(\left(C_{r}(a)_{(p)} C_{s}(b)\right)_{(q)} i_{t, w}(w+t)^{-r+2 \Delta_{a}-k-n-2} t^{k+r-2 \Delta_{a}+p+1} w^{l+k+2 n+2+q+s-2 \Delta_{b}+r-2 \Delta_{a}}\right),
\end{aligned}
$$


and apply Lemma 3.5 to obtain

$$
\begin{aligned}
& \operatorname{Res}_{t}\left(Y_{M}\left(i_{t, w} Y\left(A\left(\frac{w t}{w+t}\right) a, t^{-1}\right) A(w) b, w^{-1}\right)(w+t)^{-k-n-2} t^{k} w^{l+k+2 n+2}\right. \\
& =\operatorname{Res}_{t} \sum_{p, q, r, s}(-1)^{p-n-1}\left(C_{r}(a)_{(p)} C_{s}(b)\right)_{(q)} i_{w, t}(w+t)^{k+r-2 \Delta_{a}+p+1} t^{n-p-1} w^{l+q+s+p+1-2 \Delta_{b}} \\
& =(-1)^{n+1} \operatorname{Res}_{t} \sum_{p}(-1)^{p} Y_{M}\left(\left(A(w+t) a_{(p)} A(w) b\right), w^{-1}\right) i_{w, t}(w+t)^{k+p+1} t^{n-p-1} w^{l+p} \\
& =(-1)^{n+1} \operatorname{Res}_{t}\left(i_{w, t} Y_{M}\left(Y\left(\left(A(w+t) a, \frac{-t}{w(w+t)}\right) A(w) b\right), w^{-1}\right)(w+t)^{k} t^{n} w^{l}\right) .
\end{aligned}
$$

Thus we are reduced to prove that

$$
\begin{aligned}
& \operatorname{Res}_{t}\left(i_{w, t} Y_{M}\left(Y\left(A(t+w) a, \frac{-t}{w(t+w)}\right) A(w) b, w^{-1}\right) w^{l}(t+w)^{k} t^{n}\right. \\
& =p(a, b) \operatorname{Res}_{t}\left(Y_{M}\left(A(w) Y(a, t) b, w^{-1}\right) i_{w, t}(w+t)^{k} t^{n} w^{l}\right),
\end{aligned}
$$

or equivalently

$$
p(a, b) A(w) Y(a, t) b=i_{w, t} Y\left(A(t+w) a, \frac{-t}{(t+w) w}\right) A(w) b,
$$

which is equation (3.7) with $A(w) b$ in place of $b$. Claim (a) follows.

Let us now check (b). We need only to check that the map $m \mapsto f_{m} \in\left(M^{\dagger}\right)^{\dagger}$ where $\left\langle f_{m}, m^{\prime}\right\rangle=\overline{\left\langle m^{\prime}, m\right\rangle}$ is a $V$-module isomorphism. The map is clearly bijective since we are assuming $\operatorname{dim} M_{n}<\infty$. Now

$$
\begin{aligned}
\left\langle\left(Y_{\left(M^{\dagger}\right)^{\dagger}}(v, z) f_{m}, m^{\prime}\right\rangle\right. & =\left\langle f_{m}, Y_{M^{\dagger}}\left(A(z) v, z^{-1}\right) m^{\prime}\right\rangle=\overline{\left\langle Y_{M^{\dagger}}\left(A(z) v, z^{-1}\right) m^{\prime}, m\right\rangle} \\
& =\overline{\left.\left\langle m^{\prime}, Y_{M}\left(A(z) A\left(z^{-1}\right) v\right), z\right) m\right\rangle} .
\end{aligned}
$$

Now use (3.8) to get

$$
\left\langle\left(Y_{\left(M^{\dagger}\right)^{\dagger}}(v, z) f_{m}, m^{\prime}\right\rangle=\overline{\left\langle m^{\prime}, Y_{M}(v, z) m\right\rangle}=\left\langle f_{Y_{M}(v . z) m}, m^{\prime}\right\rangle .\right.
$$

\section{INVARIANT HERMITIAN FORMS ON CONFORMAL VERTEX ALGEBRAS}

Let $V$ be a conformal vertex algebra. By a Hermitian form on $V$ we mean a map $(\cdot, \cdot)$ : $V \times V \rightarrow \mathbb{C}$ conjugate linear in the first argument and linear in the second, such that $\left(v_{1}, v_{2}\right)=\overline{\left(v_{2}, v_{1}\right)}$ for all $v_{1}, v_{2} \in V$.

Let $\phi$ be a conjugate linear parity preserving involution of $V$. Consider the conjugate linear operator $(\mathrm{cf}(\underline{3.3}))$

$$
g=\left((-1)^{L_{0}} \sigma^{1 / 2}\right)^{-1} \phi
$$

By (3.4), we have that $g^{2}=I$. Obviously $g$ satisfies the hypothesis of Definition 3.2, Two instances of such a situation are the following.

(1) Recall from Remark 2.2 that, if $V$ is a vertex operator superalgebra, then $(-1)^{2 \Delta_{a}}=$ $(-1)^{p(a)}$ for all $a \in V$. Set $s(a)=\Delta_{a}+\frac{1}{2} p(a)$ and note that in this case $s(a)$ is an 
integer. Then

$$
\begin{aligned}
\Delta_{a}+2 \Delta_{a}^{2} & =s(a)-\frac{1}{2} p(a)+2\left(s(a)-\frac{1}{2} p(a)\right)^{2} \\
& =s(a)-\frac{1}{2} p(a)+2 s(a)^{2}-2 s(a) p(a)+\frac{1}{2} p(a)^{2} .
\end{aligned}
$$

As $p(a)-p(a)^{2}=0$ and $p(a), s(a)$ are integers, we see that

$$
\Delta_{a}+2 \Delta_{a}^{2} \equiv s(a) \quad \bmod 2
$$

so that

$$
g(a)=e^{-\pi \sqrt{-1}\left(\Delta_{a}+\frac{1}{2} p(a)\right)} \phi(a)=(-1)^{s(a)} \phi(a)=(-1)^{\Delta_{a}+2 \Delta_{a}^{2}} \phi(a)
$$

hence, if $V$ is a vertex operator superalgebra,

$$
g=(-1)^{L_{0}+2 L_{0}^{2}} \phi
$$

(2) The vertex algebra of symplectic bosons provides an example of a conformal vertex algebra that is not a vertex operator superalgebra, where our definition applies. Let $R_{\mathbb{R}}$ be a real finite dimensional even vector space equipped with a bilinear nondegenerate symplectic form $\langle\cdot, \cdot\rangle$. Let $R=\mathbb{C} \otimes R_{\mathbb{R}}$. Equip $R$ with the structure of a nonlinear conformal algebra with $\lambda$-bracket given by

$$
\left[a_{\lambda} b\right]=\langle a, b\rangle .
$$

Let $V$ be the corresponding universal enveloping vertex algebra. The Virasoro vector is

$$
L=\frac{1}{2} \sum: T\left(a^{i}\right) a_{i}:
$$

with $\left\{a_{i}\right\},\left\{a^{i}\right\}$ dual bases of $R$. The elements in $R$ are primary of conformal weight $\frac{1}{2}$. Let $\phi(r)=\bar{r}$, where $\bar{r}$ is complex conjugation with respect to $R_{\mathbb{R}}$. Then, clearly,

$$
\left[\phi(a)_{\lambda} \phi(b)\right]=\overline{\langle a, b\rangle}
$$

hence $\phi$ extends to a conjugate linear involution of $V$. In this case

$$
g(r)=-\sqrt{-1} \bar{r}, r \in R .
$$

The following definition first appeared in [3] for vertex operator superalgebras, generalizing the definition, given in [7, for vertex operator algebras.

Definition 4.1. Let $\phi$ be a conjugate linear involution of a conformal vertex algebra $V$. Choose $g$ as in Definition 3.2 and define $A(z)$ by (3.6). A Hermitian form $(\cdot, \cdot)$ on $V$ is said to be $\phi$-invariant if, for all $a \in V$,

$$
(v, Y(a, z) u)=\left(Y\left(A(z) a, z^{-1}\right) v, u\right), \quad u, v \in V .
$$

Remark 4.2. If $v \in V$ is quasi-primary, then, due to (3.14), (4.3) becomes

$$
\left(v, a_{n} u\right)=\left(g(a)_{-n}(v), u\right), \quad u, v \in V .
$$

The statement of the main result of [15] can be extended to our setting as follows.

Theorem 4.3. In the setting of Definition 4.1, the space of $\phi$-invariant Hermitian forms on $V$ is linearly isomorphic to the set of conjugate linear functionals $F \in V_{0}^{\dagger}$ such that $\left\langle F, L_{1} V_{1}\right\rangle=0$ and $\langle F, g(v)\rangle=\overline{\langle F, v\rangle}$ for all $v \in V_{0}$. 
The proof is the same as in [15, Theorem 3.1]. In the following we simply check that the argument also works in our modified setting. Recall that an element $m$ in a $V$-module $M$ is called vacuum-like if $a_{(n)} m=0$ for all $n \geq 0$ and all $a \in V$. By Proposition 2.3 of [15], a vector $m \in M$ is vacuum-like if and only if $L_{-1} m=0$, i.e. the space of vacuum-like vectors is the space $M^{L_{-1}}$ of $L_{-1}$-invariants; moreover, if $m$ is a vacuum-like vector in $M$, then $Y(u, z) m=e^{z L_{-1}} u_{(-1)} m$.

Consider the map

$$
\Psi: \operatorname{Hom}_{V}(V, M) \rightarrow M, \quad \Psi(\psi)=\psi(\mathbf{1}) .
$$

By Proposition 3.4 of [15], for any $V$-module $M, \Psi$ is an isomorphism between $\operatorname{Hom}_{V}(V, M)$ and the space $M^{L_{-1}}$.

Proof of Theorem 4.3. Assume that $(\cdot, \cdot)$ is a $\phi$-invariant Hermitian form on $V$. Note that, since $g(L)=L$, (4.4) implies that $\left(L_{0} v, w\right)=\left(v, L_{0} w\right)$. In particular the eigenspaces of $L_{0}$ are orthogonal. Define $F \in V_{0}^{\dagger}$ by $\langle F, v\rangle=(v, \mathbf{1})$. Then $(\cdot, \cdot)$ is uniquely determined by $F$, since, letting $u=1$ and taking $\operatorname{Res}_{z} z^{-1}$ of both sides of (4.3), we obtain

$$
(v, a)=\operatorname{Res}_{z} z^{-1}\left(Y\left(A(z) a, z^{-1}\right) v, \mathbf{1}\right) .
$$

By Remark 4.2,

$$
\left(L_{1} v, \mathbf{1}\right)=\left(v, L_{-1} \mathbf{1}\right)=0,\left(\mathbf{1}, L_{1} v\right)=\left(L_{-1} \mathbf{1}, v\right)=0,
$$

hence, since $L_{-1} \mathbf{1}=0$, we see that $\left\langle F, L_{1} V_{1}\right\rangle=0$.

Next we prove that, if $a \in V$, then

$$
(g(a), \mathbf{1})=(\mathbf{1}, a)
$$

Since the form is Hermitian, we have $(\mathbf{1}, a)=\overline{(a, \mathbf{1})}$, so that (4.6) implies $\langle F, g(a)\rangle=\overline{\langle F, a\rangle}$. To prove (4.6) we observe that, since $g(L)=L, g$ preserves the $L_{0}$-eigenspace decomposition. Since the eigenspaces of $L_{0}$ are orthogonal, we have that (4.6) is satisfied if $\Delta_{a} \neq 0$. We can therefore assume that $\Delta_{a}=0$, so that

$$
\begin{aligned}
(\mathbf{1}, a)=\operatorname{Res}_{z} z^{-1}(\mathbf{1}, Y(a, z) \mathbf{1}) & =\operatorname{Res}_{z} z^{-1}\left(Y\left(A(z) a, z^{-1}\right) \mathbf{1}, \mathbf{1}\right) \\
& =\sum_{r}\left(\frac{1}{r !}\left(L_{1}^{r} g(a)\right)_{\Delta_{a}} \mathbf{1}, \mathbf{1}\right) \\
& =\sum_{r} \frac{1}{r !}\left(\left(L_{1}^{r} g(a)\right)_{0} \mathbf{1}, \mathbf{1}\right) .
\end{aligned}
$$

By (4.5), in order to prove (4.6), we need only to prove that

$$
\left(L_{1}^{r} g(a)\right)_{0} \mathbf{1} \in L_{1} V_{1}, r \geq 1, a \in V_{0} .
$$

We prove by induction on $r$ that

$$
\left(L_{1}^{r} b\right)_{0} \mathbf{1} \in L_{1} V_{1}, r \geq 1, b \in V_{0} .
$$

If $r=1$, then

$$
\left[L_{1}, b_{-1}\right]=\sum_{j \in \mathbb{Z}_{+}}\left(\begin{array}{l}
2 \\
j
\end{array}\right)\left(L_{(j)} b\right)_{0}=\left(L_{-1} a\right)_{0}+2 \Delta_{b} b_{0}+\left(L_{1} b\right)_{0} .
$$

Since $\Delta_{b}=0,\left(L_{-1} b\right)_{0}=0$, so

$$
\left(L_{1} b\right)_{0} \mathbf{1}=L_{1}\left(a_{-1} \mathbf{1}\right)-b_{-1}\left(L_{1} \mathbf{1}\right)=L_{1}\left(b_{-1} \mathbf{1}\right) \in L_{1} V_{1} .
$$


If $r>1$, then

$$
\begin{aligned}
{\left[L_{1},\left(L_{1}^{r-1} b\right)_{-1}\right] } & =\sum_{j \in \mathbb{Z}_{+}}\left(\begin{array}{l}
2 \\
j
\end{array}\right)\left(L_{(j)}\left(L_{1}^{r-1} b\right)\right)_{0} \\
& =L_{-1}\left(L_{1}^{r-1} b\right)_{0}-2(r-1)\left(L_{1}^{r-1} b\right)_{0}+\left(L_{1}^{r} b\right)_{0} \\
& =-(r-1)\left(L_{1}^{r-1} b\right)_{0}+\left(L_{1}^{r} b\right)_{0}
\end{aligned}
$$

so

$$
\begin{aligned}
\left(L_{1}^{r} b\right)_{0} \mathbf{1} & =L_{1}\left(\left(L_{1}^{r-1} b\right)_{-1} \mathbf{1}\right)-\left(L_{1}^{r-1} b\right)_{-1} L_{1} \mathbf{1}+(r-1)\left(L_{1}^{r-1} b\right)_{0} \mathbf{1} \\
& =L_{1}\left(\left(L_{1}^{r-1} b\right)_{-1} \mathbf{1}\right)+(r-1)\left(L_{1}^{r-1} b\right)_{0} \mathbf{1} .
\end{aligned}
$$

The claim now follows by the induction hypothesis.

We now prove the converse statement. Consider $V$ as a $\Gamma / \mathbb{Z}$-graded vertex algebra with $\Gamma=\mathbb{Z}$ and the trivial grading $\Upsilon(\mathbb{Z})=V$. Then the state-field correspondence defines on $V$ the structure of a $\Upsilon$-twisted positive energy module. Since $\Upsilon$ is clearly compatible with $\phi$, by Thoerem 3.6, we have a $\Upsilon$-twisted module structure on $V^{\dagger}$. Fix $F \in V_{0}^{\dagger}$ which vanishes on $L_{1} V_{1}$. Then $F$ is a vacuum-like vector in $V^{\dagger}$. In particular the map $\Phi_{F}: V \rightarrow V^{\dagger}$ defined by $\Phi_{F}(v)=v_{(-1)}^{\dagger} F$ is a $V$-module homomorphism. Here and in what follows we write for simplicity $a_{n}^{\dagger}$ instead of $a_{n}^{V^{\dagger}}$. Define

$$
(u, v)=\left\langle v_{(-1)}^{\dagger} F, u\right\rangle=\left\langle\Phi_{F}(v), u\right\rangle .
$$

Let us check that this form is $\phi$-invariant:

$$
\begin{aligned}
(v, Y(a, z) u) & =\left\langle\Phi_{F}(Y(a, z) u), v\right\rangle \\
& =\left\langle Y_{V^{\dagger}}(a, z) \Phi_{F}(u), v\right\rangle \\
& =\left\langle\Phi_{F}(u), Y\left(A(z) a, z^{-1}\right) v\right\rangle \\
& =\left(Y\left(A(z) a, z^{-1}\right) v, u\right) .
\end{aligned}
$$

It remains to show that, if $\langle F, a\rangle=\overline{\langle F, g(a)\rangle}$, then the form is Hermitian. Since the form is $\phi$-invariant, by (4.6),

$$
\overline{(a, \mathbf{1})}=\overline{\langle F, a\rangle}=\langle F, g(a)\rangle=(g(a), \mathbf{1})=(\mathbf{1}, a) .
$$

We can now check that the form is Hermitian:

$$
\begin{aligned}
\overline{(u, v)} & =\operatorname{Res}_{z} z^{-1} \overline{(u, Y(v, z) \mathbf{1})} \\
& =\operatorname{Res}_{z} z^{-1} \overline{\left(Y\left(A(z) v, z^{-1}\right) u, \mathbf{1}\right)} \\
& =\operatorname{Res}_{z} z^{-1}\left(\mathbf{1}, Y\left(A(z) v, z^{-1}\right) u\right) \\
& =\operatorname{Res}_{z} z^{-1}\left(Y\left(A(z) A\left(z^{-1}\right) v, z\right) \mathbf{1}, u\right) \\
& =\operatorname{Res}_{z} z^{-1}(Y(v, z) \mathbf{1}, u)=(v, u),
\end{aligned}
$$

where, in the last step, we used (3.8).

Remark 4.4. Note that we didn't use in the proof the assumptions that $V_{0}=\mathbb{C} 1$ and that $\operatorname{dim} V_{n}=0$ for $n<0$. However, if $V_{0}=\mathbb{C} 1$, then Theorem 4.3 implies that there exists a non-zero $\phi$-invariant Hermitian form on $V$ if and only if $V_{1}$ consists of quasiprimary elements, and for this form $(\mathbf{1}, \mathbf{1}) \neq 0$. The last statement follows observing that the eigenspaces of $L_{0}$ are orthogonal to each other and the kernel of a $\phi$-invariant Hermitian form is an ideal. 
So if $(\mathbf{1}, \mathbf{1})=0$, then $\mathbf{1}$ lies in the kernel, hence the kernel of the form is $V$. Also, such a Hermitian form, satisfying $(\mathbf{1}, \mathbf{1})=1$, is unique.

Lemma 4.5. Let $M$ be a module over $\operatorname{sl}(2):=\operatorname{span}\{e, h, f\}$, such that $h$ is diagonalisable with finite-dimensional eigenspaces and negative eigenvalues. Then $M$ is a direct sum of Verma modules.

Proof. Since the sum of $h$-eigenspaces with eigenvalues congruent mod 2 is a submodule, we may assume that all eigenvalues of $h$ are congruent mod 2. Since the $h$-eigenspaces are finite-dimensional, $U$ decomposes as the direct sum of the generalized eigenspaces for the Casimir operator $\Omega$ of $\operatorname{sl}(2)$. We can therefore assume that $\Omega$ has only one eigenvalue. Any irreducible subquotient of $M$ has negative highest weight, say $n$, and the eigenvalue of $\Omega$ is $\frac{1}{2} n^{2}+n$ on it. Hence if two irreducible subquotients with non-equal highest weights have the same $\Omega$-eigenvalue, the sum of these highest weights is -2 . Since all eigenvalues of $h$ are negative and congruent mod 2 , we deduce that all irreducible subquotients have the same highest weight $n$. So, on the space $M^{e}$ of $e$-invariants (which is non-zero since the set of eigenvalues of $h$ is bounded above), $h$ has one eigenvalue $n$, and the same is true for any quotient of $M$. But on $N=M / U(\operatorname{sl}(2)) M^{e}, h$ has eigenvalues strictly smaller that $n$, hence $N^{e}=0$ and $M=U\left(s l_{2}\right) M^{e}$. Since $n<0$, any vector from $M^{e}$ generates an irreducible Verma module, so $M$ is a direct sum of Verma modules with highest weight $n$.

Proposition 4.6. Let $V$ be a conformal vertex algebra such that $L_{1} V_{1}=0$, i.e. $V_{1}$ consists of quasiprimary vectors. Let $\left\{v_{1}, v_{2}, \ldots\right\}$ be a minimal system of strong generators, which includes $L$, and consists of eigenvectors for $L$. Then, summing to the $v_{i}$ elements from $L_{-1} V$, we can make these generators quasiprimary.

Proof. By Lemma 4.5, applied to $U=\bigoplus_{n>0} V_{n}$ and $f=L_{-1}, h=-2 L_{0}, e=-\frac{1}{2} L_{1}$, we get

$$
V=\mathbb{C} \mathbf{1} \oplus \sum_{i} M_{i}
$$

where the $M_{i}$ are Verma modules for $s l(2)$ with highest weight vectors quasiprimary elements. We proceed by induction on the conformal weight of a generator. If the conformal weight is $\frac{1}{2}$ or 1 there is nothing to prove. Take now a generator $v_{i}$ whose conformal weight is strictly greater than 1 . By (4.8), we can write $v_{i}=v_{i}^{\prime}+L_{-1} b$, where $v_{i}^{\prime}$ is quasiprimary and non-zero, due to minimality. By inductive assumption $b$ lies in the subalgebra generated by quasiprimary generators. Hence we can replace $v_{i}$ by $v_{i}^{\prime}$.

Recall (cf. [11) that, since $V_{0}=\mathbb{C} \mathbf{1}$, one can define the expectation value $\langle v\rangle$ of $v$ by the equation $P_{V_{0}}(v)=\langle v\rangle \mathbf{1}$ where $P_{V_{0}}$ is the projection onto $V_{0}$ with respect to the decomposition $V=V_{0} \oplus\left(\sum_{n \neq 0} V_{n}\right)$.

Corollary 4.7. Suppose that $V$ is a conformal vertex algebra such that $V_{1}$ consists of quasiprimary vectors. Let $\phi$ be a conjugate linear involution of $V$. Then there exists a unique $\phi-$ invariant Hermitian form $(\cdot, \cdot)$ on $V$ such that $(\mathbf{1}, \mathbf{1})=1$. Moreover for any collection $\left\{U^{i} \mid i \in I\right\}$ of quasiprimary elements that strongly generate $V$ (it exists by Proposition 4.6) we have

$$
\begin{aligned}
& \left(\left(U_{j_{1}}^{i_{1}}\right)^{m_{1}} \cdots\left(U_{j_{t}}^{i_{t}}\right)^{m_{t}} \mathbf{1},\left(U_{j_{1}^{\prime}}^{i_{1}^{\prime}}\right)^{m_{1}^{\prime}} \cdots\left(U_{j_{r}^{\prime}}^{i_{r}^{\prime}}\right)^{m_{r}^{\prime}} \mathbf{1}\right) \\
& =\left\langle\left(\left(g\left(U^{i_{t}}\right)_{-j_{t}}\right)^{m_{t}} \cdots\left(g\left(U^{i_{1}}\right)_{-j_{1}}\right)^{m_{1}}\left(U_{j_{1}^{\prime}}^{i_{1}^{\prime}}\right)^{m_{1}^{\prime}} \cdots\left(U_{j_{r}^{\prime}}^{i_{r}^{\prime}}\right)^{m_{r}^{\prime}} \mathbf{1}\right\rangle .\right.
\end{aligned}
$$


Proof. Since $L_{1} V_{1}=\{0\}$, the first statement follows from Theorem 4.3 ,

To prove the second statement, note that, by (4.4), for a quasiprimary element $U$, we have $\left(g(U)_{n} v, w\right)=\left(v, U_{-n} w\right)$ and

$$
\left(U_{n} v, w\right)=\overline{\left(w, U_{n} v\right)}=\overline{\left(g(U)_{-n} w, v\right)}=\left(v, g(U)_{-n} w\right) .
$$

Since $V_{0}=\mathbb{C} \mathbf{1}$, we have $(\mathbf{1}, a)=\langle a\rangle$, hence formula (4.9) follows.

Definition 4.8. If the Hermitian form (4.9) is positive definite, the vertex algebra $V$ is called unitary.

Lemma 4.9. Let $V$ be a conformal vertex algebra and let $\phi$ be a conjugate linear involution on $V$. If there is a $\phi$-invariant positive definite Hermitian form on $V$ and $a \in V$ is a non-zero quasiprimary element such that $\phi(a)=a$ then

$$
\begin{array}{ll}
\left\langle a_{\Delta_{a}} a_{-\Delta_{a}} \mathbf{1}\right\rangle \in \mathbb{R} \backslash\{0\} & \text { if }(-1)^{2 L_{0}} \sigma(a)=a, \\
\left\langle a_{\Delta_{a}} a_{-\Delta_{a}} \mathbf{1}\right\rangle \in \sqrt{-1} \mathbb{R} \backslash\{0\} & \text { if }(-1)^{2 L_{0}} \sigma(a)=-a .
\end{array}
$$

Proof. Since

$$
(a, a)=e^{-\frac{\pi}{2} \sqrt{-1}\left(2 \Delta_{a}+p(a)\right)}\left\langle a_{\Delta_{a}} a_{-\Delta_{a}} \mathbf{1}\right\rangle,
$$

and $(a, a)>0$, we see that $\left\langle a_{\Delta_{a}} a_{-\Delta_{a}} \mathbf{1}\right\rangle$ is real and non-zero if $(-1)^{2 \Delta_{a}} \sigma(a)=a$, while it is purely imaginary and non-zero otherwise.

In conclusion of this section we discuss invariant Hermitian forms on tensor products of vertex algebras. Recall from [11] that if $V, W$ are vertex algebras, their tensor product is the vertex algebra having $V \otimes W$ as space of states, $1 \otimes \mathbf{1}$ as vacuum vector and $T \otimes I+I \otimes T$ as translation operator. The state-field correspondence is given by

$$
Y(u \otimes v, z)=Y(u, z) \otimes Y(v, z) .
$$

If $V, W$ are conformal vertex algebras, also $V \otimes W$ is conformal: its Virasoro vector is $L=$ $L_{V} \otimes \mathbf{1}+\mathbf{1} \otimes L_{W}$.

Let $\phi_{V}, \phi_{W}$ be conjugate linear involutions of $V, W$ and set

$$
\begin{gathered}
g_{V}=\left((-1)^{\left(L_{V}\right)_{0}} \sigma_{V}^{1 / 2}\right)^{-1} \phi_{V}, \quad g_{W}=\left((-1)^{\left(L_{W}\right)_{0}} \sigma_{W}^{1 / 2}\right)^{-1} \phi_{W}, \\
g=g_{V} \otimes g_{W}, \quad \phi=\phi_{V} \otimes \phi_{W} .
\end{gathered}
$$

Observe that

$$
\phi=(-1)^{L_{0}} \sigma^{1 / 2} g
$$

Moreover

$$
A(z)=e^{z L_{1}} z^{-2 L_{0}} g=\left(e^{z\left(L_{V}\right)_{1}} \otimes e^{z\left(L_{W}\right)_{1}}\right)\left(z^{-2\left(L_{V}\right)_{0}} \otimes z^{-2\left(L_{W}\right)_{0}}\right)\left(g_{V} \otimes g_{W}\right)=A_{V}(z) \otimes A_{W}(z) .
$$

If $(., .)_{V},(., .)_{W}$ are invariant Hermitian forms on $V, W$, respectively, we can induce an invariant Hermitian form $(., .)_{V \otimes W}$ on $V \otimes W$ by setting

$$
\left(v_{1} \otimes w_{1}, v_{2} \otimes w_{2}\right)_{V \otimes W}=\left(v_{1}, v_{2}\right)_{V}\left(w_{1}, w_{2}\right)_{W} .
$$

Indeed,

$$
\begin{aligned}
& \left(v_{1} \otimes v_{2}, Y(a \otimes b, z)\left(w_{1} \otimes w_{2}\right)\right)=\left(v_{1}, Y(a, z) w_{1}\right)_{V}\left(v_{2}, Y(b, z)\left(w_{2}\right)\right)_{W} \\
& =\left(Y\left(A_{V}(z) a, z^{-1}\right) v_{1}, w_{1}\right)_{V}\left(Y\left(A_{W}(z) b, z^{-1}\right) v_{2}, w_{2}\right)_{W} \\
& =\left(Y\left(A_{V}(z) \otimes A_{W}(z)(a \otimes b), z^{-1}\right)\left(v_{1} \otimes v_{2}\right), w_{1} \otimes w_{2}\right) \\
& =\left(Y\left(A(z)\left(v_{1} \otimes v_{2}\right), w_{1} \otimes w_{2}\right) .\right.
\end{aligned}
$$




\section{EXAMPles of INVARIANT HeRmitian FORMS}

In this Section we apply Corollary 4.7 to fermionic, bosonic, affine, and lattice vertex algebras.

5.1. Superfermions. Consider a superspace $A=A_{\overline{0}} \oplus A_{\overline{1}}$ endowed with a non-degenerate even skew-supersymmetric bilinear form $(. \mid$.$) . Let V(A)$ be the universal vertex algebra of the Lie conformal superalgebra $A \oplus \mathbb{C} K$ with $\lambda$-bracket

$$
\left[a_{\lambda} b\right]=(a \mid b) K
$$

$K$ being an even central element. Let $F$ be the fermionic vertex algebra:

$$
F=V(A) /(K-\mathbf{1}) .
$$

Let $\phi$ be a conjugate linear involution of $A$ such that

$$
(\phi(a) \mid \phi(b))=\overline{(a \mid b)} .
$$

By setting $\phi(K)=K$ we can extend $\phi$ to a conjugate linear involution of $A \oplus \mathbb{C} K$. Indeed

$$
\left[\phi(a)_{\lambda} \phi(b)\right]=(\phi(a) \mid \phi(b))=\overline{(a \mid b)} K=\phi((a \mid b) K) .
$$

This implies that $\phi$ extends to a conjugate linear involution of $V(A)$, hence, since $\phi(K-\mathbf{1})=$ $K-\mathbf{1}$, to an involution of $F$.

Fix a basis $\left\{a^{i}\right\}$ of $A$ and let $\left\{b^{i}\right\}$ be its dual basis w.r.t. (.|.) (i.e. $\left(a^{i} \mid b^{j}\right)=\delta_{i, j}$ ). The Virasoro vector is 11

$$
L=\frac{1}{2} \sum_{i=1}^{n}:\left(T b^{i}\right) a^{i}: .
$$

It is easy to see that $\phi(L)=L$. We embed $A$ in $F$ by identifying $v$ with $: v \mathbf{1}:$ It is easily checked that $v \in A$ is a primary element of $F$ of conformal weight $1 / 2$. Set

$$
g_{A}=\left((-1)^{L_{0}} \sigma^{1 / 2}\right)^{-1} \phi .
$$

By (3.4), we have that $g_{A}^{2}=I$. Note that

$$
g_{A}(a)=-\sqrt{-1} \phi(a), a \in A_{\overline{0}}, \quad g_{A}(a)=-\phi(a), a \in A_{\overline{1}} .
$$

The set $\left\{a^{i}\right\}$ strongly and freely generates $F$. This means that, if we order $\left(-\frac{1}{2}-\mathbb{Z}_{+}\right) \times$ $\{1, \ldots, m+n\}$ lexicographically, then the set

$$
B=\bigcup_{r}\left\{\left(a_{j_{1}}^{i_{1}}\right)^{h_{1}} \cdots\left(a_{j_{r}}^{i_{r}}\right)^{h_{r}} \mathbf{1} \mid\left(j_{1}, i_{1}\right)<\cdots<\left(j_{r}, i_{r}\right), h_{s}=1 \text { if } p\left(a^{i_{s}}\right)=1\right\}
$$

is a basis of $F$. With this choice one easily checks that

$$
F_{0}=\mathbb{C} 1, \quad F_{1}=\operatorname{span}_{\mathbb{C}}\left(\left\{: a^{i} a^{j}:\right\}\right) .
$$

Since, by Wick formula [1],

$$
\begin{aligned}
{\left[L_{\lambda}: a^{i} a^{j}:\right] } & =: T\left(a^{i}\right) a^{j}:+: a^{i} T\left(a^{j}\right):+\lambda: a^{i} a^{j}:+\int_{0}^{\lambda}\left(\left[T\left(a^{i}\right)_{\mu} a^{j}\right]+\frac{1}{2} \lambda\left[a_{\mu}^{i} a^{j}\right]\right) d \mu \\
& =T\left(: a^{i} a^{j}:\right)+\lambda: a^{i} a^{j}:-\frac{1}{2} \lambda^{2}\left(a^{i} \mid a^{j}\right)+\frac{1}{2} \lambda^{2}\left(a^{i} \mid a^{j}\right) \\
& =T\left(: a^{i} a^{j}:\right)+\lambda: a^{i} a^{j}:
\end{aligned}
$$


we see that $L_{1}\left(F_{1}\right)=\{0\}$, hence Corollary 4.7 applies. Let $(\cdot, \cdot)$ be the unique invariant Hermitian form on $F$ such that $(\mathbf{1}, \mathbf{1})=1$. By (5.3) and (4.4), the invariance amounts to

$$
\left(v_{j} a, b\right)=-\sqrt{-1}\left(a, \phi(v)_{-j} b\right), v \in A_{\overline{0}}, \quad\left(v_{j} a, b\right)=-\left(a, \phi(v)_{-j} b\right), v \in A_{\overline{1}}
$$

for all $a, b \in F, j \in \frac{1}{2}+\mathbb{Z}$.

We now discuss the unitarity of $F$. Assume that $F$ is unitary and $A_{\overline{0}} \neq 0$. Choose $a \neq 0$ in $A_{\overline{0}}$; we can assume $\phi(a)=a$. Then, by Lemma 4.9, $\left\langle a_{1 / 2} a_{-1 / 2} \mathbf{1}\right\rangle \neq 0$ but $\left\langle a_{1 / 2} a_{-1 / 2} \mathbf{1}\right\rangle=$ $(a \mid a)=0$. It follows that, if $F$ is unitary, then $A=A_{\overline{1}}$. Set $A_{\mathbb{R}}=\{a \in A \mid \phi(a)=-a\}$. Then, if $a \in A_{\mathbb{R}}$,

$$
0<(a, a)=\left\langle a_{1 / 2} a_{-1 / 2} \mathbf{1}\right\rangle=(a \mid a),
$$

so $(. \mid .)_{A_{\mathbb{R}} \times A_{\mathbb{R}}}$ must be positive definite. In such a case, choose $\left\{a^{i}\right\}$ to be an orthonormal basis of $A_{\mathbb{R}}$. It can be checked (say by induction on $r$ ) that

$$
\left\langle a_{-j_{t}}^{i_{t}} \cdots a_{-j_{1}}^{i_{1}} a_{j_{1}^{\prime}}^{i_{1}^{\prime}} \cdots a_{j_{r}^{\prime}}^{i_{r}^{\prime}} \mathbf{1}\right\rangle=\delta_{r, t} \prod_{s=1}^{r} \delta_{i_{s}, i_{s}^{\prime}} \prod_{s=1}^{r} \delta_{j_{s}, j_{s}^{\prime}}
$$

so the invariant Hermitian form is the form defined by declaring the basis $B$ to be orthonormal. Hence $F$ is a unitary conformal vertex algebra if and only if $A$ is purely odd.

5.2. Superbosons. Let $\mathfrak{h}$ be a vector superspace equipped with a supersymmetric even bilinear form $(. \mid$.$) . Let V(\mathfrak{h})$ be the universal vertex algebra of the Lie conformal superalgebra $\mathfrak{h} \oplus \mathbb{C} K$ with $\lambda$-bracket

$$
\left[v_{\lambda} w\right]=\lambda(v \mid w) K
$$

$K$ being an even central element. Let $M(\mathfrak{h})$ be the vertex algebra:

$$
M(\mathfrak{h})=V(\mathfrak{h}) /(K-\mathbf{1}) .
$$

Let $\phi$ be a conjugate linear involution of $\mathfrak{h}$. As in the previous example, if

$$
(\phi(a) \mid \phi(b))=\overline{(a \mid b)} \text {. }
$$

we can extend $\phi$ to a conjugate linear involution of $M(\mathfrak{h})$.

Fix a basis $\left\{a^{i}\right\}$ of $\mathfrak{h}$ and let $\left\{b^{i}\right\}$ be its dual basis w.r.t. (.|.) (i.e. $\left.\left(a^{i} \mid b^{j}\right)=\delta_{i, j}\right)$. The Virasoro vector is

$$
L=\frac{1}{2} \sum_{i=1}^{n}: b^{i} a^{i}:
$$

It is easy to see that $\phi(L)=L$.

We embed $\mathfrak{h}$ in $M(\mathfrak{h})$ by identifying $h$ with : $h \mathbf{1}:$. It is easily checked that $h \in \mathfrak{h}$ is a primary element of $M(\mathfrak{h})$ of conformal weight 1 .

Set

$$
g_{\mathfrak{h}}=\left((-1)^{L_{0}} \sigma^{1 / 2}\right)^{-1} \phi .
$$

By (3.4), we have that $g_{\mathfrak{h}}^{2}=I$. Note that

$$
g_{\mathfrak{h}}(a)=-\phi(a), a \in A_{\overline{0}}, \quad g_{\mathfrak{h}}(a)=\sqrt{-1} \phi(a), a \in A_{\overline{1}} .
$$

As in the previous example we can apply Corollary 4.7, thus there is a unique $\phi$-invariant Hermitian form $(\cdot, \cdot)$ on $M(\mathfrak{h})$ such that $(\mathbf{1}, \mathbf{1})=1$.

We now discuss the unitarity of $M(\mathfrak{h})$. Assume that $M(\mathfrak{h})$ is unitary and $\mathfrak{h}_{\overline{1}} \neq 0$. Choose $h \neq 0$ in $\mathfrak{h}_{\overline{1}}$; we can assume $\phi(h)=h$. Then, by Lemma 4.9, $\left\langle h_{1} h_{-1} \mathbf{1}\right\rangle \neq 0$ but $\left\langle h_{1} h_{-1} \mathbf{1}\right\rangle=$ $(h \mid h)=0$. It follows that, if $M(\mathfrak{h})$ is unitary, then $\mathfrak{h}=\mathfrak{h}_{\overline{0}}$. If this is the case, set $\mathfrak{h}_{\mathbb{R}}=\{h \in$ $\mathfrak{h} \mid \phi(h)=-h\}$. Then, as in Subsection 5.1, we must have that $(. \mid .)_{\mathfrak{h}_{\mathbb{R}} \times \mathfrak{h}_{\mathbb{R}}}$ is positive definite. 
We choose an orthonormal basis $\left\{a^{i}\right\}$ of $\mathfrak{h}_{\mathbb{R}}$, ; the $\phi$-invariant Hermitian form is therefore given by

$$
\left(\left(a_{j_{1}}^{i_{1}}\right)^{m_{1}} \cdots\left(a_{j_{t}}^{i_{t}}\right)^{m_{t}} \mathbf{1},\left(a_{j_{1}^{\prime}}^{i_{1}^{\prime}}\right)^{m_{1}^{\prime}} \cdots\left(a_{j_{r}^{\prime}}^{i_{r}^{\prime}}\right)^{m_{r}^{\prime}} \mathbf{1}\right)=\left\langle\left(a_{-j_{t}}^{i_{t}}\right)^{m_{t}} \cdots\left(a_{-j_{1}}^{i_{1}}\right)^{m_{1}}\left(a_{j_{1}^{\prime}}^{i_{1}^{\prime}}\right)^{m_{1}^{\prime}} \cdots\left(a_{j_{r}^{\prime}}^{i_{r}^{\prime}}\right)^{m_{r}^{\prime}} \mathbf{1}\right\rangle .
$$

If we order $(-\mathbb{N}) \times\{1, \ldots, \operatorname{dim} \mathfrak{h}\}$ lexicographically, then the set

$$
B=\bigcup_{r}\left\{a_{j_{1}}^{i_{1}} \cdots a_{j_{r}}^{i_{r}} \mathbf{1} \mid\left(j_{1}, i_{1}\right)<\cdots<\left(j_{r}, i_{r}\right)\right\}
$$

is a basis of $M(\mathfrak{h})$. As in Example 5.1, one can check that the basis $B$ is orthogonal; moreover the norm of each element is positive, so $M(\mathfrak{h})$ is a unitary vertex operator superalgebra, if and only if $\mathfrak{h}$ is purely even.

5.3. Affine vertex algebras. Let $\mathfrak{g}$ be a simple Lie algebra or a basic classical simple finite-dimensional Lie superalgebra and let $(. \mid$.) be a supersymmetric non-degenerate even invariant bilinear form on $\mathfrak{g}$.

We normalize the form $(. \mid$.$) on \mathfrak{g}$ by choosing an even highest root $\theta$ of $\mathfrak{g}$ as in [13] or [1, and requiring $(\theta \mid \theta)=2$. If $\mathfrak{g}=D(2,1, a)$, we assume $a \in \mathbb{R}$.

Let $\phi$ be a conjugate linear involution of $\mathfrak{g}$. We assume that

$$
(\phi(x) \mid \phi(y))=\overline{(x \mid y)}
$$

noting that, if $\mathfrak{g}$ is a Lie algebra, then the above assumption always holds.

Let Cur $\mathfrak{g}=\mathfrak{g} \oplus \mathbb{C} K$ be the current Lie conformal algebra associated to $\mathfrak{g}$ [11]. We extend $\phi$ to Cur $\mathfrak{g}$ by setting $\phi(K)=K$. Since

$$
\left[\phi(x)_{\lambda} \phi(y)\right]=[\phi(x), \phi(y)]+\lambda\left(\phi(x) \mid \phi(y) K=\phi([x, y])+\lambda \overline{(x \mid y)} K=\phi\left(\left[x_{\lambda} y\right]\right),\right.
$$

$\phi$ is a conjugate linear involution of $\operatorname{Cur} \mathfrak{g}$, hence we can extend $\phi$ to a conjugate linear involution of the universal enveloping vertex algebra $V(\mathfrak{g})$ of Cur $\mathfrak{g}$.

Choosing $k \in \mathbb{R}$, we note that $\phi(K-k \mathbf{1})=K-k \mathbf{1}$, so $\phi$ pushes down to a conjugate linear involution of the the universal affine vertex algebra of level $k$.

We identify $a \in \mathfrak{g}$ with $: a \mathbf{1}: \in V^{k}(\mathfrak{g})$. Let $h^{\vee}$ be the dual Coxeter number of $\mathfrak{g}$, i.e. the eigenvalue of the Casimir operator $\sum_{i} b^{i} a^{i}$ on $\mathfrak{g}$ divided by 2 , where $\left\{a^{i}\right\}$ and $\left\{b^{i}\right\}$ are dual bases of $\mathfrak{g}$, i. e. $\left(a^{i} \mid b^{j}\right)=\delta_{i j}$.

A Virasoro vector is provided by the Sugawara construction (defined for $k \neq-h^{\vee}$ ), see e.g. [11]:

$$
L^{\mathfrak{g}}=\frac{1}{2\left(k+h^{\vee}\right)} \sum_{i=1}^{\operatorname{dim} \mathfrak{g}}: b^{i} a^{i}: .
$$

It is easy to see that $\phi\left(L^{\mathfrak{g}}\right)=L^{\mathfrak{g}}$ provided that $k \in \mathbb{R}$.

Set

$$
g_{\mathfrak{g}}=\left((-1)^{L_{0}} \sigma^{1 / 2}\right)^{-1} \phi
$$

Explicitly

$$
g_{\mathfrak{g}}(a)=-\phi(a), a \in \mathfrak{g}_{\overline{0}}, \quad g_{\mathfrak{g}}(a)=\sqrt{-1} \phi(a), a \in \mathfrak{g}_{\overline{1}} .
$$

It is well known that $a \in \mathfrak{g}$ is a primary element of $V^{k}(\mathfrak{g})$ of conformal weight 1 (see e.g. [11]). Moreover, the set $\left\{a^{i}\right\}$ strongly and freely generates $V^{k}(\mathfrak{g})$. It follows that $V^{k}(\mathfrak{g})_{0}=\mathbb{C} 1$ and 
$L_{1} V^{k}(\mathfrak{g})_{1}=0$. By Corollary 4.7, there exists a unique $\phi$-invariant Hermitian form on $V^{k}(\mathfrak{g})$, given by

$$
\begin{aligned}
& \left(\left(a_{j_{1}}^{i_{1}}\right)^{m_{1}} \cdots\left(a_{j_{t}}^{i_{t}}\right)^{m_{t}} \mathbf{1},\left(a_{j_{1}^{\prime}}^{i_{1}^{\prime}}\right)^{m_{1}^{\prime}} \cdots\left(a_{j_{r}^{\prime}}^{i_{r}^{\prime}}\right)^{m_{r}^{\prime}} \mathbf{1}\right) \\
& =\left\langle\left(g_{\mathfrak{g}}\left(a^{i_{t}}\right)_{-j_{t}}\right)^{m_{t}} \cdots\left(g_{\mathfrak{g}}\left(a^{i_{1}}\right)_{-j_{1}}\right)^{m_{1}}\left(a_{j_{1}^{\prime}}^{i_{1}^{\prime}}\right)^{m_{1}^{\prime}} \cdots\left(a_{j_{r}^{\prime}}^{i_{r}^{\prime}}\right)^{m_{r}^{\prime}} \mathbf{1}\right\rangle .
\end{aligned}
$$

If $k \neq-h^{\vee}$, the vertex algebra $V^{k}(\mathfrak{g})$ has a unique simple quotient that we denote by $V_{k}(\mathfrak{g})$. We now discuss the unitarity of $V_{k}(\mathfrak{g})$. Assume that there is a conjugate linear involution $\phi$ such that the corresponding $\phi$-invariant form on $V_{k}(\mathfrak{g})$ is positive definite. If $\mathfrak{g}$ is not a Lie algebra then there is $a \in \mathfrak{g}_{\overline{1}}, a \neq 0$. Since $\phi$ is parity preserving we can assume $\phi(a)=a$. Then

$$
(a, a)=\left(a_{-1} \mathbf{1}, a_{-1} \mathbf{1}\right)=\sqrt{-1}\left\langle a_{1} a_{-1} \mathbf{1}\right\rangle=\sqrt{-1} k(a \mid a)=0 .
$$

If $V_{k}(\mathfrak{g})$ is unitary, then $a$ is in the maximal ideal of $V^{k}(\mathfrak{g})$, hence $k=0$ and $V_{k}(\mathfrak{g})=\mathbb{C}$.

Assume now that $\mathfrak{g}$ is a Lie algebra. Since $\phi$ is a conjugate linear involution of $V^{k}(\mathfrak{g})$ then $\phi_{\mid \mathfrak{g}}$ is a conjugate linear involution of $\mathfrak{g}$. Let $\mathfrak{g}_{\mathbb{R}}$ be the corresponding real form. As shown above, if $a \in \mathfrak{g}_{\mathbb{R}}$, then

$$
0<(a, a)=\left(a_{-1} \mathbf{1}, a_{-1} \mathbf{1}\right)=-\left\langle a_{1} a_{-1} \mathbf{1}\right\rangle=-k(a \mid a),
$$

hence $\left.(. \mid)\right|_{.\mathfrak{g}_{\mathbb{R}} \times \mathfrak{g}_{\mathbb{R}}}$ is either positive or negative definite. Let $\stackrel{\circ}{\omega}_{0}$ be a compact conjugate linear involution of $\mathfrak{g}$ such that $\phi \stackrel{\circ}{\omega}_{0}=\stackrel{\circ}{\omega}_{0} \phi$. Let $\mathfrak{k}_{\mathbb{R}}$ be the corresponding compact real form. Then

$$
\mathfrak{g}_{\mathbb{R}}=\mathfrak{g}_{\mathbb{R}} \cap \mathfrak{k}_{\mathbb{R}} \oplus \mathfrak{g}_{\mathbb{R}} \cap\left(\sqrt{-1} \mathfrak{k}_{\mathbb{R}}\right) .
$$

Since $(. \mid \cdot)_{\mid \mathfrak{k}_{\mathbb{R}} \times \mathfrak{k}_{\mathbb{R}}}$ is negative definite and $\mathfrak{k}_{\mathbb{R}} \cap \mathfrak{g}_{\mathbb{R}} \neq\{0\}$, we see that $(. \mid \cdot)_{\mathfrak{g}_{\mathbb{R}} \times \mathfrak{g}_{\mathbb{R}}}$ is negative definite so $\phi=\stackrel{\circ}{\omega}_{0}$. Let $\omega_{0}$ be the conjugate linear involution of the affinization $\widehat{\mathfrak{g}}$ of $\mathfrak{g}$ which extends $\stackrel{\circ}{\omega}_{0}$ as in $\S 7.6$ of [10]. Then the $\stackrel{\circ}{\omega}_{0}$-invariant Hermitian form on $V^{k}(\mathfrak{g})$ is defined by the property that

$$
\left(a_{j} x, y\right)=-\left(x, \stackrel{\circ}{\omega}_{0}(a)_{-j} y\right), a \in \mathfrak{g} .
$$

It follows from Theorem 11.7 of 10 combined with the formula for $\omega_{0}$ given at page 103 of loc. cit., that the $\stackrel{\circ}{\omega}_{0}$-invariant Hermitian form on $V^{k}(\mathfrak{g})$ is positive semi-definite if and only if $k \in \mathbb{Z}_{+}$.

5.4. Lattice vertex algebras. Let $Q$ be a positive definite integral lattice and $V_{Q}$ be its associated lattice vertex superalgebra (see e.g. [11, §5.4]). Set $\mathfrak{h}=\mathbb{C} \otimes_{\mathbb{Z}} Q$. Recall that the free bosons vertex operator algebra $M(\mathfrak{h})$ embeds in $V_{Q}=\oplus_{\alpha \in Q}\left(M(\mathfrak{h}) \otimes \mathbb{C} e^{\alpha}\right)$ with parity $p\left(M(\mathfrak{h}) \otimes e^{\alpha}\right)=(\alpha \mid \alpha) \bmod 2$. Let $\left\{a^{1}, \ldots, a^{l}\right\}$ be an orthogonal basis of $\mathbb{R} \otimes_{\mathbb{Z}} Q$ and let $\left\{b^{1}, \ldots, b^{l}\right\}$ be the dual basis of $\mathfrak{h}$ with respect to the form $(. \mid$.$) linearly extended from the$ form on $Q$. The Virasoro vector of $V_{Q}$ is

$$
L=\frac{1}{2} \sum_{i=1}^{l}: a^{i} b^{i}: .
$$

There are primary elements $e^{\alpha} \in V_{Q}, \alpha \in Q$ of conformal weight $\frac{1}{2}(\alpha \mid \alpha)$, such that a basis of $V_{Q}$ is

$$
B=\bigcup_{r, \alpha}\left\{a_{j_{1}}^{i_{1}} \cdots a_{j_{r}}^{i_{r}} e^{\alpha} \mid\left(j_{1}, i_{1}\right)<\cdots<\left(j_{r}, i_{r}\right)\right\},
$$

where, as in Example [5.2, $(-\mathbb{N}) \times\{1, \ldots, \operatorname{dim} \mathfrak{h}\}$ is ordered lexicographically. 
Following [7, we define a conjugate linear involution $\phi$ of $V_{Q}$ by setting

$$
\phi\left(a_{-j_{1}}^{i_{1}} \cdots a_{-j_{r}}^{i_{r}} e^{\alpha}\right)=(-1)^{r} a_{-j_{1}}^{i_{1}} \cdots a_{-j_{r}}^{i_{r}} e^{-\alpha} .
$$

It is immediate to see that $\phi(L)=L$. Since the conformal weight of $e^{\alpha}$ is $\frac{1}{2}(\alpha \mid \alpha)$, we have that $(-1)^{2 L_{0}} \sigma=I$ so, if $g=\left((-1)^{L_{0}} \sigma^{1 / 2}\right)^{-1} \phi$, then

$$
g=(-1)^{L_{0}+2 L_{0}^{2}} \phi .
$$

We have

$$
\left(V_{Q}\right)_{0}=\mathbb{C} 1, \quad\left(V_{Q}\right)_{1}=\operatorname{span}_{\mathbb{C}}\left(\left\{a^{i}\right\} \cup\left\{e^{\alpha} \mid(\alpha \mid \alpha)=2\right\}\right) .
$$

Since the $a^{i}$, as well as the $e^{\alpha}$, are primary, we see that Corollary 4.7 applies. In particular the explicit expression for the $\phi$-invariant Hermitian form is

$$
\left(\left(a_{j_{1}}^{i_{1}}\right)^{m_{1}} \cdots\left(a_{j_{t}}^{i_{t}}\right)^{m_{t}} e^{\alpha},\left(a_{j_{1}^{\prime}}^{i_{1}^{\prime}}\right)^{m_{1}^{\prime}} \cdots\left(a_{j_{r}^{\prime}}^{i_{r}^{\prime}}\right)^{m_{r}^{\prime}} e^{\beta}\right)=\delta_{\alpha,-\beta}\left\langle\left(\left(a_{-j_{t}}^{i_{t}}\right)^{m_{t}} \cdots\left(a_{-j_{1}}^{i_{1}}\right)^{m_{1}}\left(a_{j_{1}^{\prime}}^{i_{1}^{\prime}}\right)^{m_{1}^{\prime}} \cdots\left(a_{j_{r}^{\prime}}^{i_{r}^{\prime}}\right)^{m_{r}^{\prime}} \mathbf{1}\right\rangle .\right.
$$

As in Example 5.2 one can check that the basis $B$ is orthogonal and consists of elements of positive norm, so $V_{Q}$ is unitary.

\section{INVARIANT HERMITIAN FORMS ON MODULES}

Let $V$ be a conformal vertex algebra. Recall from (3.6) the definition of $A(z)$. We let

$$
\omega(v)=A(1) v, v \in V .
$$

Assume that $V$ is $\Gamma / \mathbb{Z}$-graded and let $\Upsilon$ be a $\Gamma / \mathbb{Z}$-grading compatible with $\phi$.

Proposition 6.1. $\omega\left(J_{\Upsilon}\right) \subseteq J_{-\Upsilon}$ so $\omega$ induces a conjugate linear anti-isomorphism of associative algebras $\omega: Z h u_{\Upsilon}(V) \rightarrow Z h u_{-\Upsilon}(V)$. Moreover $\omega^{2}=I d$.

Proof. By (2.18), we have

$$
\omega\left(\sum_{j \in \mathbb{Z}_{+}}\left(\begin{array}{c}
\gamma_{a} \\
j
\end{array}\right) a_{(-2+\chi(a, b)+j)} b\right)=\operatorname{Res}_{w}\left(w^{-2+\chi(a, b)} A(z)\left(Y\left((1+w)^{\gamma_{a}} a, w\right) b\right)_{\mid z=1}\right) .
$$

By (3.2)

$$
\begin{aligned}
& p(a, b) A(z) Y\left((1+w)^{\gamma_{a}} a, w\right) b= \\
& =p(a, b) e^{z L_{1}} z^{-2 L_{0}} g Y\left((1+w)^{\gamma_{a}} a, w\right) b \\
& =e^{z L_{1}} z^{-2 L_{0}} Y\left((1+w)^{\gamma_{a}} g(a),-w\right) g(b) .
\end{aligned}
$$

By 3.9

$$
e^{z L_{1}} z^{-2 L_{0}} Y\left((1+w)^{\gamma_{a}} g(a), w\right) g(b)=e^{z L_{1}} Y\left((1+w)^{\gamma_{a}} z^{-2 L_{0}} g(a),-w / z^{2}\right) z^{-2 L_{0}} g(b) .
$$

By (3.11)

$$
\begin{aligned}
& e^{z L_{1}} Y\left((1+w)^{\gamma_{a}} z^{-2 L_{0}} g(a),-w / z^{2}\right) z^{-2 L_{0}} g(b) \\
& =Y\left(e^{(z+w) L_{1}}(z+w)^{-2 L_{0}}(1+w)^{\gamma_{a}} g(a), \frac{-w}{z(z+w)}\right) e^{z L_{1}} z^{-2 L_{0}} g(b),
\end{aligned}
$$

which means that

$$
\begin{aligned}
& p(a, b) A(z) Y\left((1+w)^{\gamma_{a}} a, w\right) b= \\
& =Y\left(e^{(z+w) L_{1}}(z+w)^{-2 L_{0}}(1+w)^{\gamma_{a}} g(a), \frac{-w}{z(z+w)}\right) A(z) b,
\end{aligned}
$$


so that, since the grading is compatible with $\phi$ and $g(L)=L$,

$$
\begin{aligned}
& \left(p(a, b) A(z) Y\left((1+w)^{\gamma_{a}} a, w\right) b\right)_{\mid z=1}=Y\left(e^{(1+w) L_{1}}(1+w)^{-L_{0}+\epsilon_{a}} g(a), \frac{-w}{(1+w)}\right) \omega(b) \\
& =Y\left(e^{(1+w) L_{1}}(1+w)^{-L_{0}+\epsilon} g(a), \frac{-w}{(1+w)}\right) \omega(b)
\end{aligned}
$$

Note that

$$
e^{(1+w) L_{1}}(1+w)^{-L_{0}+\epsilon}=(1+w)^{-L_{0}+\epsilon} e^{L_{1}} .
$$

Indeed, if $a \in V$,

$$
\begin{aligned}
& e^{(1+w) L_{1}}(1+w)^{-L_{0}+\epsilon} a=e^{(1+w) L_{1}}(1+w)^{-\Delta_{a}+\epsilon_{a}} a=(1+w)^{-\Delta_{a}+\epsilon_{a}} \sum_{r \geq 0}(1+w)^{r} \frac{1}{r !} L_{1}^{r} a \\
& =\sum_{r \geq 0}(1+w)^{-\Delta_{a}+r+\epsilon_{a}} \frac{1}{r !} L_{1}^{r} a=(1+w)^{-L_{0}+\epsilon} \sum_{r \geq 0} \frac{1}{r !} L_{1}^{r} a \\
& =(1+w)^{-L_{0}+\epsilon} e^{L_{1}} a .
\end{aligned}
$$

Hence,

$$
\begin{aligned}
& \left(p(a, b) A(z) Y\left((1+w)^{L_{0}+\epsilon} a, w\right) b\right)_{\mid z=1}=Y\left((1+w)^{-L_{0}+\epsilon} e^{L_{1}} g(a), \frac{-w}{(1+w)}\right) \omega(b) \\
& =Y\left((1+w)^{-L_{0}+\epsilon} \omega(a), \frac{-w}{(1+w)}\right) \omega(b)=(1+w)^{-L_{0}} Y\left((1+w)^{\epsilon} \omega(a),-w\right)(1+w)^{L_{0}} \omega(b) .
\end{aligned}
$$

Set

$$
\varpi_{a}= \begin{cases}-\epsilon_{a}-1 & \text { if } \epsilon_{a} \neq 0, \\ 0 & \text { if } \epsilon_{a}=0 .\end{cases}
$$

Note that $\varpi$ is the function $\epsilon$ defined in Section 2 corresponding to the grading $-\Upsilon$.

Since $\epsilon_{a}+\epsilon_{b} \in \mathbb{Z}$, we have that $\varpi_{a}=-\chi(a, b)-\epsilon_{a}$ and $\chi(a, b)=1$ if and only if $\varpi_{a}+\varpi_{b} \leq$ -1 . It follows that

$$
\begin{aligned}
& \operatorname{Res}_{w}\left(w^{-2+\chi(a, b)}(1+w)^{-L_{0}} Y\left((1+w)^{\epsilon} a,-w\right)(1+w)^{L_{0}} b\right) \\
& =\operatorname{Res}_{w}\left(w^{-2+\chi(a, b)} \sum_{n, j}(-1)^{n}\left(\begin{array}{c}
-\Delta_{a}+\epsilon_{a}+n+1 \\
j
\end{array}\right)\left(a_{(n)} b\right) w^{-n-1+j}\right) \\
& =\sum_{j}(-1)^{j}\left(\begin{array}{c}
-\Delta_{a}+\epsilon_{a}+j+\chi(a, b)-1 \\
j
\end{array}\right)\left(a_{(-2+\chi(a, b)+j)} b\right) \\
& =\sum_{j}\left(\begin{array}{c}
\Delta_{a}-\epsilon_{a}-\chi(a, b) \\
j
\end{array}\right)\left(a_{(-2+\chi(a, b)+j)} b\right) \\
& =\sum_{j}\left(\begin{array}{c}
\Delta_{a}+\varpi_{a} \\
j
\end{array}\right)\left(a_{(-2+\chi(a, b)+j)} b\right)=\operatorname{Res}_{w}\left(w^{-2+\chi(a, b)} Y\left((1+w)^{L_{0}+\varpi} a, w\right) b .\right.
\end{aligned}
$$

Since $\Upsilon$ is compatible with $\phi$, we have that $\epsilon_{\omega(a)}=\epsilon_{a}$ (hence $\chi(a, b)=\chi(\omega(a), \omega(b))$. We find that

$$
\begin{aligned}
& \operatorname{Res}_{w}\left(w^{-2+\chi(a, b)} A(z)\left(Y\left((1+w)^{L_{0}} a, w\right) b\right)_{\mid z=1}\right) \\
& =p(a, b) \operatorname{Res}_{w}\left(w^{-2+\chi(\omega(a), \omega(b))} Y\left((1+w)^{L_{0}+\varpi} \omega(a), w\right) \omega(b)\right),
\end{aligned}
$$

hence, by (6.2), $\omega\left(J_{\Upsilon}\right) \subset J_{-\Upsilon}$. 
Next we prove that $\omega$ is an anti-automorphism. If $a \in V_{\Upsilon}(\mathrm{cf}$. (2.17) $)$ then $\epsilon_{a}=\epsilon_{\omega(a)}=0$, thus, if $a, b \in V_{\Upsilon}$, by (6.3),

$$
\begin{aligned}
& p(a, b) \omega(a * b)=p(a, b) \operatorname{Res}_{w}\left(w^{-1} A(z)\left(Y\left((1+w)^{L_{0}} a, w\right) b\right)_{\mid z=1}\right) \\
& =\operatorname{Res}_{w} w^{-1}(1+w)^{-L_{0}} Y(\omega(a),-w)(1+w)^{L_{0}} \omega(b) .
\end{aligned}
$$

Now use skew-symmetry $Y(a, z) b=p(a, b) e^{z L_{-1}} Y(b,-z) a$ (see e.g. [11]) to get

$$
\begin{aligned}
& \omega(a * b) \\
& =\operatorname{Res}_{w}\left(w^{-1}(1+w)^{-L_{0}} e^{-w L_{-1}} Y\left((1+w)^{L_{0}} \omega(b), w\right) \omega(a)\right) \\
& \left.=\operatorname{Res}_{w} \sum_{n, j, r}(-1)^{r}\left(\begin{array}{c}
-\Delta_{\omega(a)}+n+1-r \\
j
\end{array}\right) \frac{1}{r !} L_{-1}^{r}\left(\omega(b)_{(n)} \omega(a)\right) w^{-n-2+j+r}\right) \\
& =\sum_{j, r}(-1)^{r}\left(\begin{array}{c}
-\Delta_{\omega(a)}+j \\
j
\end{array}\right) \frac{1}{r !} L_{-1}^{r}\left(\omega(b)_{(-1+j+r)} \omega(a)\right) \\
& =\sum_{r, j}(-1)^{r}\left(\begin{array}{c}
-\Delta_{\omega(a)}+j \\
j
\end{array}\right)\left(\begin{array}{c}
-\Delta_{\omega(b)}-\Delta_{\omega(a)}+j+r \\
r
\end{array}\right)\left(\omega(b)_{(-1+j+r)} \omega(a)\right. \\
& =\sum_{r, j}(-1)^{r+j}\left(\begin{array}{c}
\Delta_{\omega(a)}-1 \\
j
\end{array}\right)\left(\begin{array}{c}
-\Delta_{\omega(b)}-\Delta_{\omega(a)}+j+r \\
r
\end{array}\right)\left(\omega(b)_{(-1+j+r)} \omega(a)\right) \\
& =\sum_{r, j}(-1)^{r+j}\left(\begin{array}{c}
\Delta_{\omega(a)}-1 \\
j
\end{array}\right)\left(\begin{array}{c}
-\Delta_{\omega(b)}-\Delta_{\omega(a)}+j+r \\
r
\end{array}\right)\left(\omega(b)_{(-1+j+r)} \omega(a)\right) \\
& =\sum_{n \geq r}(-1)^{n}\left(\begin{array}{c}
\Delta_{\omega(a)}-1 \\
n-r
\end{array}\right)\left(\begin{array}{c}
-\Delta_{\omega(b)}-\Delta_{\omega(a)}+n \\
r
\end{array}\right)\left(\omega(b)_{(-1+n)} \omega(a)\right) \\
& =\sum_{n}(-1)^{n}\left(\begin{array}{c}
-\Delta_{\omega(b)}+n-1 \\
n
\end{array}\right)\left(\omega(b)_{(-1+n)} \omega(a)\right) \\
& =\sum_{n}\left(\begin{array}{c}
\Delta_{\omega(b)} \\
n
\end{array}\right)\left(\omega(b)_{(-1+n)} \omega(a)\right)=\omega(b) * \omega(a) .
\end{aligned}
$$

We used the fact that in $Z h u_{\Upsilon} V$ we have (cf. [5, (2.35)])

$$
\frac{1}{r !} L_{-1}^{r} a=\left(\begin{array}{c}
-\Delta_{a} \\
r
\end{array}\right) a \text {. }
$$

and the Vandermonde identity on binomial coefficients.

Finally, by (3.8),

hence $\omega^{2}=I$.

$$
\omega^{2}(a)=A(1)^{2} a=a
$$

Remark 6.2. We now make explicit the map $\omega$ in the examples dealt with in Section 4 . In general, if $a$ is quasi-primary, we have, by (6.1)

$$
\omega(a)=g(a) .
$$

(1) Let $V=F$ be the fermionic vertex algebra associated to a superspace $A$ as in Example 5.1. According to [5, Theorem 3.25], $Z h u_{L_{0}}(V)$ is the Clifford algebra of $A$, i.e. the unital associative algebra generated by $A$ with relations

$$
[a, b]=(a \mid b), \quad a, b \in A \text {. }
$$


Then, according to (6.4) and (5.3),

$$
\omega(a)=-\sqrt{-1} \phi(a), a \in A_{\overline{0}}, \quad \omega(a)=-\phi(a), a \in A_{\overline{1}} .
$$

(2) Let $V=M(\mathfrak{h})$ be the vertex algebra of superbosons associated to a superspace $\mathfrak{h}$ as in Example 5.2. According to [5, Theorem 3.25], $Z h u_{L_{0}}(V)$ is the (super)symmetric algebra of $A$. Then, according to (6.4) and (5.6),

$$
\omega(a)=-\phi(a), a \in A_{\overline{0}}, \quad \omega(a)=\sqrt{-1} \phi(a), a \in A_{\overline{1}} .
$$

(3) If $V=V^{k}(\mathfrak{g})$ (cf. Example [5.3), then $Z h u_{L_{0}}(V)=U(\mathfrak{g})$ (see e.g. [5]). Then, according to (6.4),

$$
\omega(a)=-\phi(a), a \in \mathfrak{g}_{\overline{0}}, \quad \omega(a)=\sqrt{-1} \phi(a), a \in \mathfrak{g}_{\overline{1}} .
$$

(4) If $V=V_{Q}$ is a lattice vertex algebra (cf. Example 5.4), formulas (5.8) and (6.4) give

$$
\omega\left(e^{\alpha}\right)=(-1)^{\frac{(\alpha \mid \alpha)((\alpha \mid \alpha)+1)}{2}} e^{-\alpha}, \quad \omega(h)=-\bar{h}, h \in \mathfrak{h} .
$$

Here $\bar{h}$ is the conjugatie of $h \in \mathfrak{h}$ with respect to $\mathbb{R} \otimes_{\mathbb{Z}} Q$. If $Q$ is even, $Z h u_{L_{0}}\left(V_{Q}\right)$ has been proved in [6] to be isomorphic to a generalized Smith algebra, denoted there by $\overline{A(Q)}$. The algebra $\overline{A(Q)}$ is generated by elements $E_{\alpha}, \alpha \in Q, h \in \mathfrak{h}$, and the explicit formula for the isomorphism $Z h u_{L_{0}} V_{Q} \cong \overline{A(Q)}$ given in [6. Theorem 3.4] implies that

$$
\omega\left(E_{\alpha}\right)=(-1)^{\frac{(\alpha \mid \alpha)}{2}} E_{-\alpha}, \quad \omega(h)=-\bar{h}, \quad h \in \mathfrak{h},
$$

is a conjugate linear anti-automorphism of $\overline{A(Q)}$.

Definition 6.3. Let $R$ be an associative superalgebra over $\mathbb{C}$ with a conjugate linear antiinvolution $\omega$, and let $M$ be an $R$-module. A Hermitian form $(\cdot, \cdot)$ on $M$ is called $\omega$-invariant if

$$
\left(\omega(a) m_{1}, m_{2}\right)=\left(m_{1}, a m_{2}\right), a \in R, m_{1}, m_{2} \in M .
$$

Assume for the rest of this Section that $\Gamma=\mathbb{Z}$ or $\Gamma=\frac{1}{2} \mathbb{Z}$, so that $Z h u_{\Upsilon}=Z h u_{-\Upsilon}$. The following is the natural extension of Definition 4.1 to $V$-modules.

Definition 6.4. Let $\phi$ be a conjugate linear involution of the vertex algebra $V$. A Hermitian form $(\cdot, \cdot)$ on a $\Upsilon$-twisted $V$-module $M$ is called $\phi$-invariant if, for all $v \in V$,

$$
\left(m_{1}, Y_{M}(a, z) m_{2}\right)=\left(Y_{M}\left(A(z) a, z^{-1}\right) m_{1}, m_{2}\right) \text {. }
$$

From now on we assume that the module $M$ is a positive energy module (see Definition 2.6).

Remark 6.5. The space of $\phi$-invariant Hermitian forms on $M$ is linearly isomorphic to

$$
\left\{\Theta \in \operatorname{Hom}_{V}\left(M, M^{\dagger}\right) \mid\left\langle\Theta\left(m_{1}\right), m_{2}\right\rangle=\overline{\left\langle\Theta\left(m_{2}\right), m_{1}\right\rangle}\right\}
$$

Indeed, given $\Theta: M \rightarrow M^{\dagger}$ a $V$-module homomorphism, then setting, for $m_{1}, m_{2} \in M$

$$
\left(m_{1}, m_{2}\right)_{\Theta}=\left\langle\Theta\left(m_{2}\right), m_{1}\right\rangle
$$

defines a $\phi$-invariant hermitian form on $M$. In fact

$$
\begin{aligned}
\left(m_{1}, Y_{M}(a, z) m_{2}\right)_{\Theta} & =\left\langle\Theta\left(Y_{M}(a, z) m_{2}\right), m_{1}\right\rangle=\left\langle Y_{M^{\dagger}}(a, z) \Theta\left(m_{2}\right), m_{1}\right\rangle \\
& =\left\langle\Theta\left(m_{2}\right), Y_{M}\left(A(z) v, z^{-1}\right) m_{1}\right\rangle=\left(Y_{M}\left(A(z) v, z^{-1}\right) m_{1}, m_{2}\right)_{\Theta} .
\end{aligned}
$$


Conversely, let $F: M \times M \rightarrow \mathbb{C}$ be a $\phi$-invariant hermitian form; then $\Theta_{F}: M \rightarrow M^{\dagger}$ defined by $\left\langle\Theta_{F}\left(m_{1}\right), m_{2}\right\rangle=F\left(m_{2}, m_{1}\right)$ is a $V$-homomorphism from $M$ to $M^{\dagger}$. Indeed

$$
\begin{aligned}
\left\langle\Theta_{F}\left(Y_{M}(a, z) m_{1}\right), m_{2}\right\rangle & =F\left(m_{2}, Y_{M}(a, z) m_{1}\right)=F\left(Y_{M}\left(A(z) a, z^{-1}\right) m_{2}, m_{1}\right) \\
& =\left\langle\Theta_{F}\left(m_{1}\right), Y_{M}\left(A(z) a, z^{-1}\right) m_{2}\right\rangle=\left\langle Y_{M^{\dagger}}(a, z) \Theta_{F}\left(m_{1}\right), m_{2}\right\rangle .
\end{aligned}
$$

Recall that a positive energy $\Upsilon$-twisted $V$-module $M$ is said quasi-irreducible if it is generated by $M_{0}$ and there are no non-zero submodules $N \subset M$ such that $N \cap M_{0}=\{0\}$.

By [5, Lemma 2.2], if $M$ is a positive energy $\Upsilon$-twisted $V$-module, then the map $a \mapsto$ $\left(a_{0}^{M}\right)_{\mid M_{0}}$ descends to define a $Z h u_{\Upsilon} V$-module structure on $M_{0}$.

Lemma 6.6. If $M$ is quasi-irreducible then $M^{\dagger}$ is quasi-irreducible.

Proof. Set $N=V M_{0}^{\dagger}$. Then $N^{\perp}$ is graded and $\langle F, v\rangle=0$ for all $v \in N_{0}^{\perp}, F \in M_{0}^{\dagger}$. This implies that $N_{0}^{\perp}=\{0\}$, so $N^{\perp}=\{0\}$, hence $N=M^{\dagger}$.

If $N$ is a graded submodule of $M^{\dagger}$ with $N_{0}=\{0\}$ then $N^{\perp}$ is a graded submodule of $M$ containing $M_{0}$. Since $M_{0}$ generates $M$, it follows that $N^{\perp}=M$ hence $N=\{0\}$.

Proposition 6.7. Let $M$ be a $\Upsilon$-twisted positive-energy $V$-module generated by $M_{0}$. Then the space of $\phi$-invariant Hermitian forms on $M$ is linearly isomorphic to the set of $\omega$ invariant Hermitian forms on the ZhuฯV-module $M_{0}$.

Proof. If $(\cdot, \cdot)$ is a $\phi$-invariant Hermitian form on $M$, then $(\cdot, \cdot)_{0}=(\cdot, \cdot)_{\mid M_{0} \times M_{0}}$ is a $\omega$-invariant Hermitian form on $M_{0}$ by Proposition 6.1.

Let $(\cdot, \cdot)_{0}$ be a $\omega$-invariant Hermitian form on the $Z h u_{\Upsilon} V$-module $M_{0}$. Let $N$ be the sum of all graded submodules $N^{\prime}$ of $M$ such that $N^{\prime} \cap M_{0}=\{0\}$. Then $M / N$ is quasi-irreducible and $(M / N)_{0}=M_{0}$. Define $\Phi_{0}: M_{0} \rightarrow M_{0}^{\dagger}$ by setting $\Phi_{0}\left(m_{1}\right)\left(m_{2}\right)=\left(m_{2}, m_{1}\right)_{0}$. Since the form $(\cdot, \cdot)_{0}$ is $\omega$-invariant, we have

$$
\begin{aligned}
\Phi_{0}\left(v_{0}^{M} m_{1}\right)\left(m_{2}\right) & =\left(m_{2}, v_{0}^{M} m_{1}\right)_{0}=\left(\omega(v)_{0}^{M} m_{2}, m_{1}\right)_{0}=\Phi_{0}\left(m_{1}\right)\left(\omega(v)_{0}^{M} m_{2}\right) \\
& =\left(v_{0}^{M^{\dagger}} \Phi_{0}\right)\left(m_{2}\right)\left(m_{1}\right),
\end{aligned}
$$

so $\Phi_{0}$ is a $Z h u_{\Upsilon}(V)$-module map between $M_{0}$ and $M_{0}^{\dagger}$. By Lemma 6.6 and 5, Theorem 2.30], there is a $V$-module map $\Phi: M / N \rightarrow(M / N)^{\dagger}$ such that $\Phi_{\mid M_{0}}=\Phi_{0}$. Define, for $m_{1}, m_{2} \in M$,

$$
\left(m_{1}, m_{2}\right)=\Phi\left(m_{2}+N\right)\left(m_{1}+N\right) .
$$

It is clear that the form $(\cdot, \cdot)$ is $\phi$-invariant and that $(\cdot, \cdot)_{0}=(\cdot, \cdot)_{\mid M_{0} \times M_{0}}$. It remains to check that the form is Hermitian.

Consider the form $(\cdot, \cdot)^{\prime}$ defined by $\left(m_{1}, m_{2}\right)^{\prime}=\overline{\left(m_{2}, m_{1}\right)}$. Note that $(\cdot, \cdot)^{\prime}$ is $\phi$ invariant:

$$
\begin{aligned}
\left(m_{1}, Y_{M}(a, z) m_{2}\right)^{\prime} & =\overline{\left(Y_{M}(a, z) m_{2}, m_{1}\right)}=\overline{\left(Y_{M}\left(A(z) A\left(z^{-1}\right) a, z\right) m_{2}, m_{1}\right)} \\
& =\overline{\left(m_{2}, Y_{M}\left(A(z) a, z^{-1}\right) m_{1}\right)}=\left(Y_{M}\left(A(z) a, z^{-1}\right) m_{1}, m_{2}\right)^{\prime} .
\end{aligned}
$$

Since $(\cdot, \cdot)_{0}$ is Hermitian, then

$$
(\cdot, \cdot)_{\mid M_{0} \times M_{0}}^{\prime}=(\cdot, \cdot)_{\mid M_{0} \times M_{0}}
$$

hence $(\cdot, \cdot)^{\prime}=(\cdot, \cdot)$. 
Remark 6.8. Theorem 4.3 is a consequence of Proposition 6.7. Indeed, the space of $\omega-$ invariant Hermitian forms on $V_{0}$ is linearly isomorphic to $\left(V_{0} / L_{1} V_{1}\right)^{\dagger}$. The isomorphism is defined by mapping $(\cdot, \cdot)_{0}$ to $F_{(\cdot, \cdot)_{0}}$ where $F_{(\cdot, \cdot)_{0}}(v)=(v, \mathbf{1})_{0}$. To prove that this map is well defined, let us check that $F_{(\cdot, \cdot)_{0}}\left(L_{1} V_{1}\right)=0$. If $v \in V_{1}$, then

$$
\left.L_{1} v=\left(L_{1} v\right)_{0} \mathbf{1}=\left(v_{0}+\left(L_{1} v\right)_{0}\right) \mathbf{1}\right)=\omega(g(v))_{0} \mathbf{1},
$$

so

$$
F_{(\cdot, \cdot)_{0}}\left(L_{1} v\right)=\left(L_{1} v, \mathbf{1}\right)_{0}=\left(\left(\omega(g(v))_{0} \mathbf{1}, \mathbf{1}\right)_{0}=-\left(\mathbf{1}, g(v)_{0} \mathbf{1}\right)_{0}=0\right.
$$

The inverse is the map $F \mapsto(\cdot, \cdot)_{F}$, where $(v, w)_{F}=F\left(\omega(w)_{0} v\right)$. Let us check that $(\cdot, \cdot)_{F}$ is $\omega$-invariant. If $u, v \in V_{0}$ and $w \in V_{\mathbb{Z}}$, then $\left(u, w_{0} v\right)_{F}=F\left(\omega\left(w_{0} v\right)_{0} u\right)$ and $\left(\omega(w)_{0} u, v\right)_{F}=$ $F\left(\omega(v)_{0} \omega(w)_{0} u\right)$. Viewing $F$ as an element of $V^{\dagger}$, we observe that

$$
F\left(\omega\left(w_{0} v\right)_{0} u\right)=\left(\left(w_{0} v\right)_{0}^{V^{\dagger}} F\right)(u), F\left(\omega(v)_{0} \omega(w)_{0} u\right)=\left(w_{0}^{V^{\dagger}} v_{0}^{V^{\dagger}} F\right)(u),
$$

so it is enough to check that

$$
\left(w_{0} v\right)_{0}^{V^{\dagger}} F=w_{0}^{V^{\dagger}} v_{0}^{V \dagger} F
$$

Observe that, since $\left\langle F, L_{1} V_{1}\right\rangle=0, L_{-1} F=0, F$ is a vacuum-like element of $V^{\dagger}$. It follows from Proposition 3.4 of [15] that the map $\Phi: V \rightarrow V^{\dagger}$ defined by $\Phi(a)=a_{(-1)}^{V^{\dagger}} F$ is a $V$-module map. In particular,

$$
\Phi\left(a_{(n)} b\right)=a_{(n)}^{V^{\dagger}} \Phi(b)=a_{(n)}^{V^{\dagger}}\left(b_{(-1)}^{V^{\dagger}} F\right)
$$

On the other hand

$$
\Phi\left(a_{(n)} b\right)=\left(a_{(n)} b\right)_{(-1)}^{V^{\dagger}} F
$$

so

$$
a_{(n)}^{V^{\dagger}}\left(b_{(-1)}^{V^{\dagger}} F\right)=\left(a_{(n)} b\right)_{(-1)}^{V^{\dagger}} F
$$

Since $\Delta_{v}=\Delta_{w_{0} v}=0$, we find $v_{(-1)}^{V^{\dagger}} F=v_{0}^{V^{\dagger}} F$ and $\left(w_{0} v\right)_{(-1)}^{V^{\dagger}} F=\left(w_{0} v\right)_{0}^{V^{\dagger}} F$, so (6.7) follows.

\section{INVARIANT HERMITIAN FORMS ON $W$-ALGEBRAS}

We adopt the setting and notation of Section 1 of [13]. We let $W^{k}(\mathfrak{g}, x, f)$ be the universal $W$-algebra of level $k \in \mathbb{R}$ associated to the datum $(\mathfrak{g}, x, f)$, where $\mathfrak{g}$ is a simple finite-dimensional Lie superalgebra with a reductive even part and a non-zero even invariant supersymmetric bilinear form (.|.), $x$ is an ad-diagonalizable element of $\mathfrak{g}$ with eigenvalues in $\frac{1}{2} \mathbb{Z}, f$ is an even element of $\mathfrak{g}$ such that $[x, f]=-f$ and the eigenvalues of ad $x$ on the centralizer $\mathfrak{g}^{f}$ of $f$ in $\mathfrak{g}$ are non-positive. Recall that we are assuming that $a \in \mathbb{R}$ for $\mathfrak{g}=D(2,1 ; a)$. We call the datum $(\mathfrak{g}, x, f)$ a Dynkin datum if there is a $s l(2)$-triple $\{f, h, e\}$ containing $f$ and $x=\frac{1}{2} h$.

Let

$$
\mathfrak{g}=\bigoplus_{j \in \frac{1}{2} \mathbb{Z}} \mathfrak{g}_{j}
$$

be the grading of $\mathfrak{g}$ by $a d(x)$-eigenspaces. We assume that $k \neq-h^{\vee}$ so that $W^{k}(\mathfrak{g}, x, f)$ has a Virasoro vector. Then $W^{k}(\mathfrak{g}, x, f)$ is a conformal vertex algebra in the sense of Definition 2.1 .

Remark 7.1. It is easy to show that a datum $(\mathfrak{g}, x, f)$ as above is independent, up to isomorphism, from the choice of $f$, hence we may use notation $W^{k}(\mathfrak{g}, x)$. 
Remark 7.2. An important special case is when $f$ is a minimal nilpotent element of the even part of $\mathfrak{g}$, i.e. $f$ is the root vector $e_{-\theta}$ corresponding to a maximal even root $\theta$. In this case, the invariant bilinear form (.|.) is normalized so that $(\theta \mid \theta)=2$. Choose the root vector $e_{\theta} \in \mathfrak{g}_{\theta}$ in such a way that $\left(e_{\theta} \mid e_{-\theta}\right)=\frac{1}{2}$. Setting $x=\left[e_{\theta}, e_{-\theta}\right]$, it is clear that $\left(\mathfrak{g}, x, e_{-\theta}\right)$ is a Dynkin datum. Identifying the Cartan subalgebra $\mathfrak{h}$ with its dual using (.|.), one has $x=\theta / 2$. The algebra $W^{k}(\mathfrak{g}, \theta / 2)$ is called a minimal $W$-algebra.

Lemma 7.3. Let $\phi$ be a conjugate linear involution of $\mathfrak{g}$ such that

$$
\phi(f)=f, \quad \phi(x)=x .
$$

Assume also, as in Subsection 5.3, that

$$
\overline{(\phi(X) \mid \phi(Y))}=(X \mid Y)
$$

so that $\phi$ extends to a conjugate linear involution of $V^{k}(\mathfrak{g})$. Then $\phi$ descends to an involution of the vertex algebra $W^{k}(\mathfrak{g}, x, f)$.

Proof. Let $A$ be the superspace $\Pi\left(\sum_{j>0} \mathfrak{g}_{j}\right)$ where $\Pi$ is the reverse parity functor. Let $A^{*}$ be the linear dual of $A$ and set $A_{c h}=A \oplus A^{*}$. Define the form $\langle\cdot, \cdot\rangle_{c h}$ on $A_{c h}$ by setting, for $a, b \in A, a^{\prime}, b^{\prime} \in A^{*}$,

$$
\langle a, b\rangle_{c h}=\left\langle a^{\prime}, b^{\prime}\right\rangle_{c h}=0, \quad\left\langle a, b^{\prime}\right\rangle_{c h}=b^{\prime}(a), \quad\left\langle b^{\prime}, a\right\rangle_{c h}=-p\left(a, b^{\prime}\right) a^{\prime}(b) .
$$

Let $A_{n e}$ be the superspace $\mathfrak{g}_{1 / 2}$ equipped with the form $\langle\cdot, \cdot\rangle_{n e}$ defined by

$$
\langle a, b\rangle_{n e}=(f \mid[a, b]) .
$$

Since $\phi(f)=f$,

$$
\langle\phi(a), \phi(b)\rangle_{n e}=(f \mid[\phi(a), \phi(b)])=(\phi(f) \mid \phi([a, b]))=\overline{(f \mid[a, b])}=\overline{\langle a, b\rangle}_{n e} .
$$

It follows that $\phi$ extends to a conjugate linear involution of $F\left(A_{n e}\right)$. Similarly, setting $\phi\left(b^{*}\right)(a)=\overline{b^{*}(\phi(a))}$ for $b^{*} \in A^{*}$ and $a \in A$, we have

$$
\left\langle\phi(a), \phi\left(b^{*}\right)\right\rangle_{c h}=\phi\left(b^{*}\right)(\phi(a))=\overline{b^{*}(a)}={\overline{\left\langle a, b^{*}\right\rangle_{c h}}},
$$

so $\phi$ extends to a conjugate linear involution of $F\left(A_{c h}\right)$. It follows that $\phi$ is a conjugate linear involution of the vertex algebra $\mathcal{C}(\mathfrak{g}, f, x, k)=V^{k}(\mathfrak{g}) \otimes F\left(A_{c h}\right) \otimes F\left(A_{n e}\right)$.

Recall that there is an element $d \in \mathcal{C}(\mathfrak{g}, f, x, k)$ such that $d_{0}$ is an odd derivation and $d_{0}^{2}=0$, making $\mathcal{C}(\mathfrak{g}, f, x, k)$ a complex. It is easy to see that $\phi(d)=d$, hence the involution $\phi$ descends to an involution of the vertex algebra $W^{k}(\mathfrak{g}, x, f)=H^{0}(\mathcal{C}(\mathfrak{g}, f, x, k), d)$ [12], [13].

Recall from [13] that the vertex algebra $W^{k}(\mathfrak{g}, x, f)$ is strongly and freely generated by fields $J^{\left\{x_{i}\right\}}$ with $\left\{x_{i}\right\}$ a basis of $\mathfrak{g}^{f}$, the centralizer of $f$ in $\mathfrak{g}$. We can clearly assume that the elements $x_{i}$ are homogeneous with respect to the gradation $\mathfrak{g}^{f}=\oplus_{j} \mathfrak{g}_{j}^{f}$. Let $\mathfrak{g}_{\mathbb{R}}$ be the fixed point set of $\phi$. By (7.3), we see that $(. \mid .)_{\mathfrak{g}_{\mathbb{R}} \times \mathfrak{g}_{\mathbb{R}}}$ is a real bilinear form. Since $\phi(x)=x$, we see that $\mathfrak{g}_{j}=\left(\mathfrak{g}_{j} \cap \mathfrak{g}_{\mathbb{R}}\right) \oplus\left(\sqrt{-} 1 \mathfrak{g}_{j} \cap \mathfrak{g}_{\mathbb{R}}\right)$. Moreover $\langle\cdot, \cdot\rangle_{n e}$ is real when restricted to $\mathfrak{g}_{1 / 2} \cap \mathfrak{g}_{\mathbb{R}}$. Likewise, we can identify the real dual of $\mathfrak{g}_{+} \cap \mathfrak{g}_{\mathbb{R}}$ with the set of $b^{*} \in A^{*}$ such that $\phi\left(b^{*}\right)=b^{*}$. It follows that we can identify the algebra $\mathcal{C}\left(\mathfrak{g}_{\mathbb{R}}, f, x, k\right)$ as a real subalgebra of $\mathcal{C}(\mathfrak{g}, f, x, k)$. We can therefore carry out the construction of the fields $J^{\{a\}}$ for $a \in \mathfrak{g}_{\mathbb{R}}^{f}$ inside $\mathcal{C}\left(\mathfrak{g}_{\mathbb{R}}, f, x, k\right)$ and therefore obtain that $\phi\left(J^{\{a\}}\right)=J^{\{a\}}$. As $a \in \mathfrak{g}^{f}$ can be written as $a=a_{\mathbb{R}}+i b_{\mathbb{R}}$ with $a_{\mathbb{R}}, b_{\mathbb{R}} \in \mathfrak{g}_{\mathbb{R}}^{f}$, we see that we can construct the field $J^{\{a\}}$ so that $\phi\left(J^{\{a\}}\right)=J^{\{\phi(a)\}}$.

Let $L^{\mathfrak{g}}$ the Virasoro vector for $V^{k}(\mathfrak{g})$ defined in (5.7). The vertex algebra $W^{k}(g, x, f)$ carries a Virasoro vector $L$, making it a conformal vertex algebra, which is the homology class of $L^{\mathfrak{g}}+T(x)+L^{c h}+L^{n e}$ (see [12]). 
In particular, by the above discussion and the explicit expressions for $L^{\mathfrak{g}}, L^{c h}, L^{n e}$, we obtain that $\phi(L)=L$. Following (3.3) we set

$$
g=\left((-1)^{L_{0}} \sigma^{1 / 2}\right)^{-1} \phi .
$$

If $x_{i} \in \mathfrak{g}_{j}^{f}$, then the conformal weight of $J^{\left\{x_{i}\right\}}$ is $1-j$. It follows that

$$
W^{k}(\mathfrak{g}, x, f)_{0}=\mathbb{C} \mathbf{1}, \quad W^{k}(\mathfrak{g}, x, f)_{1}=\operatorname{span}\left(\left\{J^{\left\{x_{i}\right\}} \mid x_{i} \in \mathfrak{g}_{0}^{f}\right\}\right) .
$$

Theorem 7.4. (a) Let $v \in \mathfrak{g}_{0}^{f}$. If $J^{\{v\}} \in W^{k}(\mathfrak{g}, x, f)_{1}$ is quasiprimary for more than one $k \in \mathbb{C}$, then

$$
(x \mid v)=0 .
$$

(b) If the datum $(\mathfrak{g}, x, f)$ is a Dynkin datum, then the elements $J^{\{v\}}$ are primary for all $v \in \mathfrak{g}_{0}^{f}$ and $k \in \mathbb{C}\left(k \neq-h^{\vee}\right)$. In particular, by Corollary 4.7 , there is a unique $\phi$-invariant Hermitian form $(\cdot, \cdot)$ on $W^{k}(\mathfrak{g}, x, f)$ such that $(\mathbf{1}, \mathbf{1})=1$.

(c) Assume that $\mathfrak{g}$ is a Lie algebra. If (7.4) holds for a datum $(\mathfrak{g}, x, f)$ and all $v \in \mathfrak{g}_{0}^{f}$, then it is a Dynkin datum.

Proof. By [12, Theorem 2.4b], if $v \in \mathfrak{g}_{0}^{f}$, then

$$
\left[L_{\lambda} J^{\{v\}}\right]=(T+\lambda) J^{\{v\}}+\lambda^{2}\left(\frac{1}{2} s t r_{\mathfrak{g}_{+}}(a d v)-\left(k+h^{\vee}\right)(v \mid x)\right),
$$

hence claim (a) follows immediately.

If the datum $(\mathfrak{g}, x, f)$ is a Dynkin datum, then $2(x \mid v)=([e, f] \mid v)=(e \mid[f, v])=0$ if $v \in \mathfrak{g}^{f}$. Hence for (b) it suffices to show $\operatorname{str}_{\mathfrak{g}_{j}}(a d v)=0$ for all $j \in \frac{1}{2} \mathbb{N}$ and $v \in \mathfrak{g}_{0}^{f}$.

Consider the following bilinear form on $\mathfrak{g}_{j}$ :

$$
<a, b>=\left((a d f)^{2 j} a \mid b\right) .
$$

By $s l(2)-$ representation theory, $(a d f)^{2 j}: \mathfrak{g}_{j} \rightarrow \mathfrak{g}_{-j}$ is injective for $j>0$, hence $\langle\cdot, \cdot>$ is non-degenerate. The form is clearly $a d \mathfrak{g}_{0}^{f}$-invariant. The form is super (resp. skew-super) symmetric if $j \in \mathbb{Z}$ (resp. $\left.j \in \frac{1}{2}+\mathbb{Z}\right)$ :

$$
<a, b>=\left((a d f)^{2 j} a \mid b\right)=(-1)^{2 j}\left(a \mid(a d f)^{2 j} b\right)=(-1)^{2 j} p(a, b)<b, a>.
$$

Hence for $v \in \mathfrak{g}_{0}^{f}$, ad $v$ lies in $\operatorname{osp}\left(\mathfrak{g}_{j}\right)\left(\operatorname{resp} . \operatorname{spo}\left(\mathfrak{g}_{j}\right)\right)$ if $j \in \mathbb{Z}\left(\right.$ resp. $\left.j \in \frac{1}{2}+\mathbb{Z}\right)$. Hence in either case its supertrace is 0 . This proves (b).

By Theorem 1.1 from [8], $x=\frac{1}{2} h+c$, where $\{e, h, f\}$ is an $s l(2)$-triple for some $e \in \mathfrak{g}_{1}$ and $c$ is a semisimple central element from the centralizer of this triple. We may assume that $c$ is defined over $\mathbb{R}$. But then $(x \mid c)=\left(\frac{1}{2} h+c \mid c\right)=(c \mid c)$. Since we are assuming that $\mathfrak{g}$ is a simple Lie algebra, (7.4) implies that $c=0$, proving (c).

Remark 7.5. Let $\mathfrak{g}$ be a simple Lie algebra. It follows from Theorem 7.4 that a datum $(\mathfrak{g}, x, f)$ is Dynkin if and only if $\left(x \mid \mathfrak{g}_{0}^{f}\right)=0\left(\Longleftrightarrow\left(x \mid \mathfrak{g}^{f}\right)=0\right)$. In other words a $\frac{1}{2} \mathbb{Z}$-grading of $\mathfrak{g}$ is Dynkin iff $f \in \mathfrak{g}_{-1}$, all eigenvalues of $a d x$ on $\mathfrak{g}^{f}$ are non-positive and $\left(x \mid \mathfrak{g}^{f}\right)=0$.

Example 7.6. Let $\mathfrak{g}=\operatorname{sl}(3)$ with the data $\left(\mathfrak{g}, \frac{1}{2}\left(E_{11}-E_{33}\right), E_{31}, k\right)$ and $\left(\mathfrak{g},-2 E_{11}+E_{22}+\right.$ $\left.E_{33}, E_{31}, k\right)$. The first one is a Dynkin datum corresponding to the minimal $W$-algebra $W^{k}(\mathfrak{g}, \theta / 2)$. The second one is not Dynkin: indeed, if $v=E_{11}-2 E_{22}+E_{33}$, then $v \in \mathfrak{g}_{0}^{f}$ and $(x \mid v) \neq 0$.

Corollary 7.7. Assume that $(\mathfrak{g}, x, f)$ is a Dynkin datum. Then there is a unique $\phi$-invariant Hermitian form $(.,$.$) on W^{k}(\mathfrak{g}, x, f)$ such that $(\mathbf{1}, \mathbf{1})=1$. 
Proof. By Theorem 7.4 (b), we can apply Corollary 4.7.

We now describe the $\phi$-invariant Hermitian form more explicitly using formula (4.9). Fix a basis $\left\{x^{i}\right\}$ of $\mathfrak{g}^{f}$. Set $\Delta_{i}=\Delta_{x^{i}}$ and $p_{i}=p\left(x^{i}\right)$. By Proposition 4.6 we may assume that the fields $J^{\left\{x_{i}\right\}}$ are quasiprimary for all $i$. We can clearly assume that $\phi\left(x^{i}\right)=x^{i}$ for all $i$. Since $\phi(L)=L$, the proof of Lemma 4.5, hence of Proposition 4.6, can be done over $\mathbb{R}$, so $\phi\left(J^{\left\{x^{i}\right\}}\right)=J^{\left\{x^{i}\right\}}$ and let $J^{\left\{x^{i}\right\}}(z)=\sum_{n \in-\Delta_{i}+\mathbb{Z}} J_{n}^{\left\{x^{i}\right\}} z^{-n-\Delta_{i}}$.

Order the set

$$
\left\{(j, i) \in \frac{1}{2} \mathbb{Z}_{+} \times\left\{0, \ldots, \operatorname{dim} \mathfrak{g}^{f}-1\right\} \mid j \in \Delta_{i}+\mathbb{Z}_{+}\right\}
$$

lexicographically. Then the set

$$
\left\{\left(J_{-j_{1}}^{\left\{x^{i_{1}}\right\}}\right)^{m_{1}} \cdots\left(J_{-j_{t}}^{\left\{x^{i}\right\}}\right)^{m_{t}} \mathbf{1} \mid m_{i}=0 \text { or } 1 \text { if } x^{i} \text { is odd }\right\}
$$

is a basis of $W^{k}(\mathfrak{g}, x, f)$. Since

$$
g\left(J^{\left\{x^{i}\right\}}\right)=(-\sqrt{-1})^{2 \Delta_{i}+p_{i}} J^{\left\{x^{i}\right\}},
$$

formula (4.9) gives that

$$
\begin{aligned}
& \left(\left(J_{j_{1}}^{\left\{x^{i_{1}}\right\}}\right)^{m_{1}} \cdots\left(J_{j_{t}}^{\left\{x^{i_{t}}\right\}}\right)^{m_{t}} \mathbf{1},\left(J_{j_{1}^{\prime}}^{\left\{x_{1}^{i_{1}^{\prime}}\right\}}\right)^{m_{1}^{\prime}} \cdots\left(J_{j_{r}^{\prime}}^{\left\{x^{i^{\prime}}\right\}}\right)^{m_{t}^{\prime}} \mathbf{1}\right) \\
& =(-\sqrt{-1})^{\sum_{r} m_{r}\left(2 \Delta_{i_{r}}+p_{i_{r}}\right)}\left\langle\left(J_{-j_{t}}^{\left\{x^{i_{t}}\right\}}\right)^{m_{t}} \cdots\left(J_{-j_{1}}^{\left\{x^{i_{1}}\right\}}\right)^{m_{1}}\left(J_{j_{1}^{\prime}}^{\left\{x_{1}^{i_{1}^{\prime}}\right\}}\right)^{m_{1}^{\prime}} \cdots\left(J_{j_{r}^{\prime}}^{\left\{x_{r}^{\prime}\right\}}\right)^{m_{r}^{\prime}}\right\rangle .
\end{aligned}
$$

Remark 7.8. Set $R=\operatorname{span}\left(T^{k}\left(J^{\left\{x^{i}\right\}}\right), k \in \mathbb{Z}^{+}\right)$. Let $\pi_{Z}$ be the quotient map from $W^{k}(\mathfrak{g}, x, f)$ to $Z h u_{L_{0}}\left(W^{k}(\mathfrak{g}, x, f)\right)$. Set $\mathfrak{w}=\operatorname{span}\left(\pi_{Z}\left(J^{\left\{x^{i}\right\}}\right)\right)$. By (7.5) the set

$$
\left\{:\left(T^{k_{1}} J^{\left\{x^{i_{1}}\right\}}\right)^{m_{1}} \cdots\left(T^{k_{t}} J^{\left\{x^{i_{t}}\right\}}\right)^{m_{t}}: \mid m_{i}=0 \text { or } 1 \text { if } x^{j_{i}} \text { is odd }\right\}
$$

is a basis of $W^{k}(\mathfrak{g}, x, f)$. It follows from Theorem 3.25 of [5] that

$$
R /\left(L_{-1}+L_{0}\right) R \simeq \mathfrak{w}
$$

has the structure of a nonlinear Lie superalgebra and that $Z h u_{L_{0}}\left(W^{k}(\mathfrak{g}, x, f)\right)$ is its universal enveloping algebra. In particular the set

$$
\left\{\left(\pi_{Z} J^{\left\{x^{i_{1}}\right\}}\right)^{m_{1}} * \cdots *\left(\pi_{Z} J^{\left\{x^{i_{t}}\right\}}\right)^{m_{t}} \mid m_{i}=0 \text { or } 1 \text { if } x^{j_{i}} \text { is odd }\right\}
$$

is a basis of $Z h u_{L_{0}}\left(W^{k}(\mathfrak{g}, x, f)\right)$. Since, by Proposition 4.6, $J^{\left\{x_{i}\right\}}$ can be chosen to be quasiprimary for all $i$, it is clear that the involution $\omega$ in this basis is given by

$\omega\left(\left(\pi_{Z} J^{\left\{x^{i_{1}}\right\}}\right)^{m_{1}} * \cdots *\left(\pi_{Z} J^{\left\{x^{i_{t}}\right\}}\right)^{m_{t}}\right)=(-\sqrt{-1})^{\sum_{r} m_{r}\left(2 \Delta_{i_{r}}+p_{i_{r}}\right)}\left(\pi_{Z} J^{\left\{x^{i_{t}}\right\}}\right)^{m_{t}} * \cdots *\left(\pi_{Z} J^{\left\{x^{i_{1}}\right\}}\right)^{m_{1}}$.

We now restrict to the case of a minimal $W$-algebra $W^{k}(\mathfrak{g}, \theta / 2)$ (see Remark 7.2) where one has a more explicit description of $Z h u_{L_{0}}\left(W^{k}(\mathfrak{g}, \theta / 2)\right)$ and its involution.

Set $\mathfrak{g}^{\natural}=\mathfrak{g}_{0}^{f}$. Then $\mathfrak{g}^{f}=\mathfrak{g}^{\natural} \oplus \mathfrak{g}_{-1 / 2} \oplus \mathbb{C} f$. The elements $J^{\{v\}}$ are uniquely determined for $v \in \mathfrak{g}^{\natural} \oplus \mathfrak{g}_{-1 / 2}$ and have been computed explicitly in [12]. One usually denotes $J^{\{v\}}$ by $G^{\{v\}}$ if $v \in \mathfrak{g}_{-1 / 2}$. We also write $\mathfrak{g}^{\natural}=\oplus_{i=0}^{r} \mathfrak{g}_{i}$ with $\mathfrak{g}_{0}$ the (possibly zero) center and $\mathfrak{g}_{i}$ a simple ideal for $i>0$.

Set, for $u, v \in \mathfrak{g}_{-1 / 2}$,

$$
\langle u, v\rangle=\left(e_{\theta} \mid[u, v]\right)
$$


and note that $\langle\cdot, \cdot\rangle$ is a $\mathfrak{g}^{\natural-i n v a r i a n t ~ s k e w-s u p e r s y m m e t r i c ~ b i l i n e a r ~ f o r m ~ o n ~} \mathfrak{g}_{-1 / 2}$. Fix a basis $\left\{a_{i}\right\}$ of $\mathfrak{g}^{\natural}$ and a basis $\left\{u_{i}\right\}$ of $\mathfrak{g}_{-1 / 2}$. Then $W^{k}(\mathfrak{g}, \theta)$ has as set of free generators

$$
\left\{J^{\left\{a_{i}\right\}}\right\} \cup\left\{G^{\left\{u_{i}\right\}}\right\} \cup\{L\} .
$$

Moreover the $\lambda$-brackets between generators is known explicitly [12, [13, 1], [14, and Section $8, L$ is the Virasoro vector and its central charge is $\frac{k \text { sdimg }}{k+h^{\vee}}-6 k+h^{\vee}-4$, the $J^{\{u\}}$ are primary of conformal weight 1 , the $G^{\{v\}}$ are primary of conformal weight $\frac{3}{2}$ and

(1) $\left[J^{\{a\}}{ }_{\lambda} J^{\{b\}}\right]=J^{\{[a, b]\}}+\lambda \delta_{i j}\left(k+\frac{h^{\vee}-h_{0, i}^{\vee}}{2}\right)(a \mid b)$ for $a \in \mathfrak{g}_{i}^{\natural}, b \in \mathfrak{g}_{j}^{\natural}$;

(2) $\left[J^{\{a\}}{ }_{\lambda} G^{\{u\}}\right]=G^{\{[a, u]\}}$ for $u \in \mathfrak{g}_{-1 / 2}, a \in \mathfrak{g}^{\natural}$;

(3)

$$
\begin{aligned}
& {\left[G_{\lambda}^{\{u\}} G^{\{v\}}\right]=-2\left(k+h^{\vee}\right)\langle u, v\rangle L+\langle u, v\rangle \sum_{\alpha=1}^{\operatorname{dim} \mathfrak{g}^{\natural}}: J^{\left\{a^{\alpha}\right\}} J^{\left\{a_{\alpha}\right\}}:+} \\
& 2 \sum_{\alpha, \beta=1}^{\operatorname{dim} \mathfrak{g}^{\natural}}\left\langle\left[a_{\alpha}, u\right],\left[v, a^{\beta}\right]\right\rangle: J^{\left\{a^{\alpha}\right\}} J^{\left\{a_{\beta}\right\}}:+2(k+1)(\partial+2 \lambda) J^{\left.\left\{\left[e_{\theta}, u\right], v\right]^{\natural}\right\}} \\
& +2 \lambda \sum_{\alpha, \beta=1}^{\operatorname{dim} \mathfrak{g}^{\natural}}\left\langle\left[a_{\alpha}, u\right],\left[v, a^{\beta}\right]\right\rangle J^{\left\{\left[a^{\alpha}, a_{\beta}\right]\right\}}+2 p(k) \lambda^{2}\langle u, v\rangle .
\end{aligned}
$$

Here $\left\{a_{\alpha}\right\}$ (resp. $\left\{u_{\gamma}\right\}$ ) is a basis of $\mathfrak{g}^{\natural}\left(\right.$ resp. $\left.\mathfrak{g}_{1 / 2}\right)$ and $\left\{a^{\alpha}\right\}$ (resp. $\left\{u^{\gamma}\right\}$ ) is the corresponding dual basis w.r.t. $(. \mid$.$\left.) (resp w.r.t. \langle\cdot, \cdot\rangle_{\mathrm{ne}}=\left(e_{-\theta} \mid[\cdot, \cdot]\right)\right)$, $a^{\natural}$ is the orthogonal projection of $a \in \mathfrak{g}_{0}$ on $\mathfrak{g}^{\natural}, a_{i}^{\natural}$ is the projection of $a^{\natural}$ on the $i$ th minimal ideal $\mathfrak{g}_{i}^{\natural}$ of $\mathfrak{g}^{\natural}, k_{i}=k+\frac{1}{2}\left(h^{\vee}-h_{0, i}^{\vee}\right)$, where $h_{0, i}^{\vee}$ is the dual Coxeter number of $\mathfrak{g}_{i}^{\natural}$ with respect to the restriction of the form $(. \mid$.$) ,$ and $p(k)$ is the monic quadratic polynomial given in Table 4 of 1 . See Appendix 8 for the derivation of formula (3) from the formulas given in 12 .

Identify $\mathfrak{w}$ with $\mathfrak{g}^{\natural} \oplus \mathfrak{g}_{-1 / 2} \oplus \mathbb{C} L$ by identifying $\pi_{Z} J^{\{a\}}$ with $a, \pi_{Z} G^{\{v\}}$ with $v$ and $\pi_{z} L$ with L. As in Remark 7.8, a basis of $Z h u_{L_{0}}\left(W^{k}(\mathfrak{g}, \theta)\right)$ is given by $\left\{u_{i_{1}}^{m_{1}} * \cdots * u_{i_{t}}^{m_{t}} * a_{j_{1}}^{n_{1}} * \cdots * a_{j_{r}}^{n_{r}} * L^{k} \mid i_{1}<\cdots i_{t} ; j_{1}<\cdots<j_{r} ; m_{p}, n_{q} \in\{0,1\}\right.$ if $a_{i_{p}}$ or $u_{j_{q}}$ is odd $\}$.

Moreover the commutation relations among the generators are as follows (here $[\cdot, \cdot]_{\mathfrak{g}}$ denotes the bracket in $\mathfrak{g}$, while $[\cdot, \cdot]$ is the bracket in $Z h u_{L_{0}}\left(W^{k}(\mathfrak{g}, \theta)\right)$.

(1) $L$ is a central element,

(2) $[a, b]=[a, b]_{\mathfrak{g}}$ if $a, b \in \mathfrak{g}^{\natural}$,

(3) $[a, v]=[a, v]_{\mathfrak{g}}$ if $a \in \mathfrak{g}^{\natural}$ and $v \in \mathfrak{g}_{-1 / 2}$,

$$
\begin{aligned}
{[u, v]=} & \langle u, v\rangle\left(\sum_{\alpha=1}^{\operatorname{dim} \mathfrak{g}^{\natural}}\left(a^{\alpha} * a_{\alpha}-\left[a^{\alpha}, a_{\alpha}\right]_{\mathfrak{g}}\right)-2\left(k+h^{\vee}\right) L-\frac{1}{2} p(k)\right) \\
& +\sum_{\alpha, \beta=1}^{\operatorname{dim} \mathfrak{g}^{\natural}}\left\langle\left[a_{\alpha}, u\right]_{\mathfrak{g}},\left[v, a^{\beta}\right]_{\mathfrak{g}}\right\rangle\left(2 a^{\alpha} * a_{\beta}-\left[a^{\alpha}, a_{\beta}\right]_{\mathfrak{g}}\right) .
\end{aligned}
$$


By (2), (3) we can drop the subscript $\mathfrak{g}$ from the bracket. Moreover observe that

$$
\sum_{\alpha=1}^{\operatorname{dim} \mathfrak{g}^{\natural}}\left[a^{\alpha}, a_{\alpha}\right]_{\mathfrak{g}}=0
$$

and that

$$
2 a^{\alpha} * a_{\beta}-\left[a^{\alpha}, a_{\beta}\right]_{\mathfrak{g}}=2 a^{\alpha} * a_{\beta}-\left[a^{\alpha}, a_{\beta}\right]=a^{\alpha} * a_{\beta}+p\left(a_{\alpha}, a_{\beta}\right) a_{\beta} * a^{\alpha} .
$$

Setting $L^{\prime}=2\left(k+h^{\vee}\right) L+\frac{1}{2} p(k)$, a new generating space is $\mathfrak{g}^{\natural} \oplus \mathfrak{g}_{-1 / 2} \oplus \mathbb{C} L^{\prime}$ and the commutation relations are (1) with $L^{\prime}$ in place of $L,(2),(3)$ and

$$
[u, v]=\langle u, v\rangle\left(\sum_{\alpha=1}^{\operatorname{dim} \mathfrak{g}^{\natural}} a^{\alpha} * a_{\alpha}-L^{\prime}\right)+\sum_{\alpha, \beta=1}^{\operatorname{dim} \mathfrak{g}^{\natural}}\left\langle\left[a_{\alpha}, u\right]_{\mathfrak{g}},\left[v, a^{\beta}\right]_{\mathfrak{g}}\right\rangle\left(a^{\alpha} * a_{\beta}+p\left(a_{\alpha}, a_{\beta}\right) a_{\beta} * a^{\alpha}\right) .
$$

It is then clear that $Z h u_{L_{0}}\left(W^{k}(\mathfrak{g}, \theta / 2)\right)$ does not depend on $k$ if $k \neq-h^{\vee}$.

The involution $\omega$ is easily computed: since the generators are quasiprimary, we have by (6.4): $\omega\left(J^{\{a\}}\right)=g\left(J^{\{a\}}\right)$, hence

$$
\begin{aligned}
& \omega\left(L^{\prime}\right)=L^{\prime}, \\
& \omega(a)=(-1)^{p(a)+1}(\sqrt{-1})^{p(a)} \phi(a), a \in \mathfrak{g}^{\natural}, \\
& \omega(v)=(-1)^{p(v)}(\sqrt{-1})^{p(v)+1} \phi(v), v \in \mathfrak{g}_{-1 / 2} .
\end{aligned}
$$

Recall that, if $k+h^{\vee} \neq 0$, then $W^{k}(\mathfrak{g}, \theta / 2)$ has a unique simple quotient $W_{k}(\mathfrak{g}, \theta / 2)$. Remark that the maximal proper ideal $I^{k}$ of $W^{k}(\mathfrak{g}, \theta / 2)$ is the kernel of the invariant Hermitian form on $W^{k}(\mathfrak{g}, \theta / 2)$, hence one can induce a invariant Hermitian form on $W_{k}(\mathfrak{g}, \theta / 2)$. The latter vertex algebra is unitary if and only if the invariant form on $W^{k}(\mathfrak{g}, \theta / 2)$ is positive semidefinite. Recall from [1] that a level $k$ is collapsing for $W^{k}(\mathfrak{g}, \theta / 2)$ if $W_{k}(\mathfrak{g}, \theta / 2)$ is contained in its affine vertex algebra part.

Theorem 7.9. Assume that $W_{k}(\mathfrak{g}, \theta / 2)$ is unitary.

(1) If $\mathfrak{g} \neq \operatorname{sl}(2)$ is a Lie algebra then $k$ is a collapsing level.

(2) If $\mathfrak{g}^{\natural}$ is not a Lie algebra then $k$ is a collapsing level.

In particular, if $W_{k}(\mathfrak{g}, \theta / 2)$ unitary for three different values of $k$, then either $\mathfrak{g}=\operatorname{sl}(2)$ or $\mathfrak{g}$ is not a Lie algebra and $\mathfrak{g}^{\natural}$ is a Lie algebra.

Proof. (1). By assumption $\mathfrak{g}_{-1 / 2} \neq 0$, take a nonzero $u \in \mathfrak{g}_{-1 / 2}$ such that $\phi(u)=u$ and compute using (7.6) with $m_{1}=m_{1}^{\prime}=1$ :

$$
\left(G^{\{u\}}, G^{\{u\}}\right)=\left(G_{-3 / 2}^{\{u\}} \mathbf{1}, G_{-3 / 2}^{\{u\}} \mathbf{1}\right)=\sqrt{-1}\left\langle G_{3 / 2}^{\{u\}} G_{-3 / 2}^{\{u\}} \mathbf{1}\right\rangle=4 p(k)\langle u, u\rangle=0 .
$$

If the form on $W^{k}(\mathfrak{g}, \theta / 2)$ is positive semidefinite then $G^{\{u\}} \in I^{k}$, hence $k$ is a collapsing level.

(2). Take $a \in \mathfrak{g}^{\natural}$ such that $p(a)=1, \phi(a)=a$. Compute using (77.6) with $m_{1}=m_{1}^{\prime}=1$

$$
\left(J^{\{a\}}, J^{\{a\}}\right)=\left(J_{-1}^{\{a\}} \mathbf{1}, J_{-1}^{\{a\}} \mathbf{1}\right)=\sqrt{-1}\left\langle J_{1}^{\{a\}} J_{-1}^{\{a\}} \mathbf{1}\right\rangle=0,
$$

hence $J^{\{a\}} \in I^{k}$. Assume that $\mathfrak{g}^{\natural}$ is simple; since $I^{k} \cap \mathfrak{g}^{\natural}$ is and ideal of $\mathfrak{g}^{\natural}$, then $\mathfrak{g}^{\natural} \subset I^{k}$. Since $\mathfrak{g}_{-1 / 2}$ is not the trivial representation of $\mathfrak{g}^{\natural}$, there exist $b \in \mathfrak{g}^{\natural}$ and $u \in \mathfrak{g}_{-1 / 2}$ such that $[b, u] \neq 0$. Since $\left[J^{\{b\}}{ }_{\lambda} G^{\{u\}}\right]=G^{\{[b, u]\}}$, [1, Prop. 3.2] implies that $k$ is collapsing. 
The only remaining case, according to [1, Table 3], is $\mathfrak{g}=o \operatorname{sp}(m \mid n), m \geq 5$. In this case $\mathfrak{g}^{\natural}=\operatorname{osp}(m-4 \mid n) \oplus \operatorname{sl}(2)$ and $\mathfrak{g}_{-1 / 2}=\mathbb{C}^{m-4 \mid n} \otimes \mathbb{C}^{2}$, and the previous argument applies to $o s p(m-4 \mid n)$ acting on $\mathbb{C}^{m-4 \mid n}$.

Remark 7.10. The proof of Theorem 7.9 shows more generally that if there exists an odd (resp. even) element of integer (resp. half-integer) conformal weight in a $W$-algebra $W^{k}(\mathfrak{g}, x)$, which does not lie in the kernel of its homomorphism to $W_{k}(\mathfrak{g}, f)$, then the latter $W$-algebra is not unitary.

In general, even at collapsing levels, the simple vertex algebra $W_{k}(\mathfrak{g}, \theta / 2)$ might not be unitary. It is clear that if $W^{k}(\mathfrak{g}, \theta / 2)$ collapses to $\mathbb{C}$ then $W_{k}(\mathfrak{g}, \theta / 2)$ is unitary. The list of such cases is given in Proposition 3.4 of [1].

In the next proposition we deal with other collapsing levels allowing unitarity.

Proposition 7.11. Assume $W_{k}(\mathfrak{g}, \theta / 2) \neq \mathbb{C}$. If $k$ is a collapsing level and there is a conjugate linear involution $\phi$ on $W_{k}(\mathfrak{g}, \theta / 2)$ such that the corresponding $\phi$-invariant form is unitary, then the pair $(\mathfrak{g}, k)$ is one in the following list

$$
\begin{aligned}
\mathfrak{g} & =\operatorname{sl}(m \mid n), m \neq n, n+1, n+2, m \geq 2, & & k=-1, \\
\mathfrak{g} & =G_{2}, & & k=-4 / 3, \\
\mathfrak{g} & =\operatorname{osp}(m \mid n), m-n \geq 10, m-n \text { even, } & k & =-2, \\
\mathfrak{g} & =\operatorname{spo}(2 \mid 3), & k & =-3 / 4, \\
\mathfrak{g} & =D\left(2,1 ;-\frac{1+n}{n+2}\right), n \in \mathbb{N}, & k & =-\frac{1+n}{n+2} .
\end{aligned}
$$

Proof. Looking at [2, Table 5] one gets that in the cases listed in the statement there is a conjugate linear involution $\phi$ such that the $\phi$-invariant Hermitian form on $W_{k}(\mathfrak{g}, \theta / 2)$ is positive definite. In case (7.7) $W_{k}(\mathfrak{g}, \theta / 2)$ is $M(\mathbb{C})$ (Heisenberg vertex algebra) and its unitarity is shown in Subsection 5.2, In cases (7.8), (7.9), (7.10), (7.11), $W_{k}(\mathfrak{g}, \theta / 2)$ is a simple affine vertex algebra at positive integral level, hence unitarity follows from Subsection 5.3 .

It remains only to check that the cases in the statement are the only cases where one can have unitarity at a collapsing level $k$, but, as explained in the discussion at the end of Subsection 5.3, a simple affine vertex algebra $V_{k}(\mathfrak{g})$ can be unitary if and only if $\mathfrak{g}$ is even and $k$ is a positive integer.

Corollary 7.12. The following simple minimal $W$-algebras are unitary:

(1) $W_{-1}(s l(m \mid n), \theta / 2) \cong M(\mathbb{C}), m \neq n, n+1, n+2, m \geq 2$, where $M(\mathbb{C})$ is the Heisenberg vertex algebra with central charge $c=1$;

(2) $W_{-4 / 3}\left(G_{2}, \theta / 2\right) \cong V_{1}(\operatorname{sl}(2))$ with central charge $c=1$;

(3) $W_{-2}(\operatorname{osp}(m \mid n), \theta / 2) \cong V_{\frac{m-n-8}{2}}(\operatorname{sl}(2)), m-n \geq 10, m$ and $n$ even, with central charge $c=\frac{3(m-n-8)}{m-n-4}$

(4) $W_{-3 / 4}(\operatorname{spo}(2 \mid 3), \theta / 2) \cong V_{1}(\operatorname{sl}(2))$ with central charge $c=1$;

(5) $W_{-\frac{1+n}{n+2}}\left(D\left(2,1 ;-\frac{1+n}{n+2}\right), \theta / 2\right) \cong V_{n}(\operatorname{sl}(2))$ with central charge $c=\frac{3 n}{2+n}, n \in \mathbb{Z}_{+}$.

Remark 7.13. Case (4) of Corollary 7.12 is of special interest since $W_{k}(s p o(2 \mid 3))$, tensored with one fermion, is the $N=3$ superconformal algebra. The collapsing level corresponds to the central charge 1 of the simple W-algebra, isomorphic to $V_{1}(s l(2))$, hence to the central charge $c=3 / 2$ of the $N=3$ superconformal algebra, which is therefore unitary. This has been already observed in [16]. 
Remark 7.14. Another interesting case of Corollary 7.12 is $(5)$. Recall that $W_{k}(D(2,1 ; a)$, tensored with four fermions and one boson, is the big $N=4$ superconformal algebra [13]. It follows from Corollary 7.12 that this algebra is unitary when $a=-\frac{1+n}{n+2}, n \in \mathbb{Z}_{+}$, the central charge being $-6 a$.

\section{Appendix: $\lambda$-Brackets in minimal $W$-Algebras}

If $u \in \mathfrak{g}_{-1 / 2}$ and $v \in \mathfrak{g}_{1 / 2}$, then a direct computation shows that

$$
[u, v]=\sum_{\alpha}\left([u, v] \mid a^{\alpha}\right) a_{\alpha}+\frac{([u, v] \mid x)}{(x \mid x)} x=\sum_{\alpha}\left(a_{\alpha} \mid[u, v]\right) a^{\alpha}+\frac{(x \mid[u, v])}{(x \mid x)} x
$$

SO

$$
\begin{aligned}
& {\left[u_{\gamma}, v\right]^{\natural}=\sum_{\alpha}\left(\left[u_{\gamma}, v\right] \mid a^{\alpha}\right) a_{\alpha}=\sum_{\alpha}\left(u_{\gamma} \mid\left[v, a^{\alpha}\right]\right) a_{\alpha},} \\
& {\left[u, u^{\gamma}\right]^{\natural}=\sum_{\alpha}\left(a_{\alpha} \mid\left[u, u^{\gamma}\right]\right) a^{\alpha}=\sum_{\alpha}\left(\left[a_{\alpha}, u\right] \mid u^{\gamma}\right) a^{\alpha} .}
\end{aligned}
$$

Moreover,

$$
\left[\left[u, u^{\gamma}\right],\left[u_{\gamma}, v\right]\right]^{\natural}=\sum_{\alpha, \beta}\left(\left[a_{\alpha}, u\right] \mid u^{\gamma}\right)\left(u_{\gamma} \mid\left[v, a^{\beta}\right]\right)\left[a^{\alpha}, a_{\beta}\right]
$$

Since, if $v \in \mathfrak{g}_{-1 / 2}, v=\sum_{\gamma}\left(v \mid u^{\gamma}\right)\left[e_{-\theta}, u_{\gamma}\right]$, we obtain

$$
2\left[e_{\theta}, v\right]=2 \sum_{\gamma}\left(v \mid u^{\gamma}\right)\left[e_{\theta},\left[e_{-\theta}, u_{\gamma}\right]\right]=2 \sum_{\gamma}\left(v \mid u^{\gamma}\right)\left[x, u_{\gamma}\right]=\sum_{\gamma}\left(v \mid u^{\gamma}\right) u_{\gamma} .
$$

Substituting we find

$$
\begin{aligned}
& \sum_{\gamma}\left(\left[a_{\alpha}, u\right] \mid u^{\gamma}\right)\left(u_{\gamma} \mid\left[v, a^{\beta}\right]\right)=\left(\sum_{\gamma}\left(\left[a_{\alpha}, u\right] \mid u^{\gamma}\right) u_{\gamma} \mid\left[v, a^{\beta}\right]\right) \\
& =2\left(\left[e_{\theta},\left[a_{\alpha}, u\right]\right] \mid\left[v, a^{\beta}\right]\right)=2\left\langle\left[a_{\alpha}, u\right],\left[v, a^{\beta}\right]\right\rangle .
\end{aligned}
$$

Recall from [1], 14] that

$$
\begin{aligned}
& {\left[G^{\{u\}}{ }_{\lambda} G^{\{v\}}\right]=-2\left(k+h^{\vee}\right)\langle u, v\rangle L+\langle u, v\rangle \sum_{\alpha=1}^{\operatorname{dim} \mathfrak{g}^{\natural}}: J^{\left\{a^{\alpha}\right\}} J^{\left\{a_{\alpha}\right\}}:+} \\
& \sum_{\gamma=1}^{\operatorname{dim} \mathfrak{g}_{1 / 2}}: J^{\left\{\left[u, u^{\gamma}\right]^{\natural}\right\}} J^{\left\{\left[u_{\gamma}, v\right]^{\natural}\right\}}:+2(k+1)(\partial+2 \lambda) J^{\left\{\left[\left[e_{\theta}, u\right], v\right]^{\natural}\right\}} \\
& +\lambda \sum_{\gamma \in S_{1 / 2}} J^{\left\{\left[\left[u, u^{\gamma}\right],\left[u_{\gamma}, v\right]\right]^{\natural}\right\}}+2 p(k) \lambda^{2}\langle u, v\rangle,
\end{aligned}
$$


where $p(k)$ is a monic quadratic polynomial in $k$, listed in [1, Table 4]. Using the above formulas we can rewrite (8.1) as

$$
\begin{aligned}
& {\left[G^{\{u\}}{ }_{\lambda} G^{\{v\}}\right]=-2\left(k+h^{\vee}\right)\langle u, v\rangle L+\langle u, v\rangle \sum_{\alpha=1}^{\operatorname{dim} \mathfrak{g}^{\natural}}: J^{\left\{a^{\alpha}\right\}} J^{\left\{a_{\alpha}\right\}}:+} \\
& 2 \sum_{\alpha, \beta}\left\langle\left[a_{\alpha}, u\right],\left[v, a^{\beta}\right]\right\rangle: J^{\left\{a^{\alpha}\right\}} J^{\left\{a_{\beta}\right\}}:+2(k+1)(\partial+2 \lambda) J^{\left\{\left[\left[e_{\theta}, u\right], v\right]^{\natural}\right\}} \\
& +2 \lambda \sum_{\alpha, \beta}\left\langle\left[a_{\alpha}, u\right],\left[v, a^{\beta}\right]\right\rangle J^{\left\{\left[a^{\alpha}, a_{\beta}\right]\right\}}+2 p(k) \lambda^{2}\langle u, v\rangle .
\end{aligned}
$$

\section{REFERENCES}

[1] D. Adamović, V. G. Kac, P. Möseneder Frajria, P. Papi, O. Perše, Conformal embeddings of affine vertex algebras in minimal W-algebras I: Structural results, J. Algebra 500 (2018), 117-152.

[2] D. Adamović, V. G. Kac, P. Möseneder Frajria, P. Papi, O. Perše, An application of collapsing levels to the representation theory of affine vertex algebras, International Mathematics Research Notices 2020, Issue 13, July 2020, 4103-4143.

[3] C. Ai, X. Lin, On the unitary structures of vertex operator superalgebras, Journal of Algebra 487 (2017), 217-243.

[4] R. E. Borcherds, Vertex algebras, Kac-Moody algebras, and the Monster, Proc. Natl. Acad. Sci. USA 8 3 (1986), 3068-3071.

[5] A. De Sole, V. G. Kac, Finite vs affine W-algebras, Japan J. Math. 1 (2006), 137-261.

[6] C. Dong, H. Li, G. Mason, Certain associative algebras similar to U(sl2) and Zhu's algebra A(V $\left.V_{L}\right)$. J. Algebra 196 (1997), no. 2, 532-551.

[7] C. Dong, X. Lin, Unitary vertex operator superalgebras, J. Algebra 397 (2014), 252-277.

[8] A. G. Elashvili, V. G. Kac, Good gradings in simple Lie algebras, Amer. Math. Soc. Transl. (2) 213 (2005), 85-104.

[9] I.B. Frenkel, Yi-Zhi Huang, J. Lepowsky, On axiomatic approaches to vertex operator algebras and modules, Memoirs of the American Mathematical Society 494, 1993.

[10] V. G. Kac, Infinite dimensional Lie algebras, 3ed. Cambridge University Press, 1990.

[11] V. G. Kac, Vertex algebras for beginners, 2ed. AMS (1998).

[12] V. G. Kac, S.-S. Roan, M. Wakimoto, Quantum reduction for affine superalgebras, Comm. Math. Phys. 241 (2003) 307-342.

[13] V. G. Kac and M. Wakimoto, Quantum reduction and representation theory of superconformal algebras., Adv. in Math. 185 (2004), 400-458.

[14] V. G. Kac, P. Möseneder Frajria, P. Papi, Yangians vs minimal W-algebras: a surprizing coincidence, Comm. Contemp. Math. 23 (2021), no. 4, 2050036.

[15] H. Li, Symmetric invariant bilinear forms on vertex operator superalgebras. J. Pure and App. Algebra, 96 (1994), 279-297.

[16] A. Schwimmer, N. Seiberg, Comments on the $N=2,3,4$ superconformal algebras in two dimensions, Phys. Lett. B 184 (1987), no. 2,3, 191-196.

V.K.: Department of Mathematics, MIT, 77 Mass. Ave, Cambridge, MA 02139;

kac@math.mit .edu

P.MF.: Politecnico di Milano, Polo regionale di Como, Via Anzani 4, 22100, Como, Italy;

pierluigi.moseneder@polimi.it

P.P.: Dipartimento di Matematica, Sapienza Università di Roma, P.le A. Moro 2, 00185, Roma, Italy; papi@mat.uniroma1.it 